\title{
Pairing-specific, Activity-dependent Presynaptic Facilitation at Aplysia Sensory-Motor Neuron Synapses in Isolated Cell Culture
}

\author{
Lise S. Eliot, Robert D. Hawkins, Eric R. Kandel, and Samuel Schacher \\ Center for Neurobiology and Behavior, College of Physicians and Surgeons of Columbia University, New York, New York \\ 10032
}

\begin{abstract}
Synapses made by Aplysia sensory neurons onto motor- and interneuron followers in the intact nervous system exhibit an associative form of synaptic facilitation that is thought to contribute to classical conditioning of the animal's gill and siphon withdrawal reflex (Hawkins et al., 1983; Walters and Byrne, 1983). Here we demonstrate that a similar associative facilitation can be induced between individual sensory and motor neurons isolated in culture. Pairing tetanic stimulation with either of two facilitatory transmitters, $5-\mathrm{HT}$ or small cardioactive peptide, considerably prolongs facilitation compared to either tetanus or transmitter alone. When corrected for the depression that occurs simply in response to lowfrequency testing, the facilitation produced by one pairing trial does not decay for more than $\mathbf{2 0}$ min after training. This facilitation requires the temporal pairing $(0.5 \mathrm{sec}$ forward interstimulus interval) of the two stimuli, tetanus and 5-HT. Delivering the same two stimull in an unpaired fashion (1 min forward interval) fails to produce the long-lasting effect. Measurements of spontaneous transmitter release during either paired or unpaired training reveal no changes in unitary mEPSP or mEPSC ("mini") amplitude, indicating that the facilitation involves a presynaptic mechanism. While both forms of training dramatically increase the initial frequency of spontaneous release, mini frequency does not remain elevated as long as the evoked EPSP following paired training, nor does paired training specifically enhance spontaneous release trequency. Pairing-specific facilitation was not blocked by the protein kinase $\mathrm{C}$ inhibitor $\mathrm{H} 7$. In contrast, the same training procedure produced pairing-specific increases of sensory neuron excitability and action potential width, suggesting that CAMP-mediated processes are involved in the paired effect. Although $\mathrm{Ca}^{2+}$ influx is necessary for the associative effect (Abrams, 1985), we find that the facilitation does not require influx through L-type voltagegated $\mathrm{Ca}^{2+}$ channels, since the effect was not blocked by the dihydropyridine antagonist nitrendipine. Together, these findings indicate that the mechanism underlying associative, activity-dependent facilitation is intrinsic to the sensory neuron synapse, that it is presynaptically mediated by process-
\end{abstract}

\footnotetext{
Received Feb. 26, 1993; revised July 7, 1993; accepted July 13, 1993.

We thank Tracey Cohen for technical assistance, and Sarah Mack, Charles Lam, and Robert Wooley for help in preparing the figures. This work was supported by the Howard Hughes Mcdical Institute (E.R.K.), Grant MH 26212 from the NIMH (R.D.H.), Grant GM 32099 from the NIH (S.S.), and an NSF predoctoral fellowship (L.S.E.).

Correspondence should be addressed to Lise Eliot, Division of Neuroscience, Baylor College of Medicine, One Baylor Plaza, Houston, TX 77030

Copyright (C) 1994 Society for Neuroscience $0270-6474 / 94 / 140368-16 \$ 05.00 / 0$
}

es unique to evoked synaptic transmission, and that it appears to involve a pairing-specific broadening of the presynaptic action potential, allowing enhenced $\mathrm{Ca}^{2+}$ influx through the dihydropyridine-insensitive channels responsible for release.

IKey words: synaptic plasticity, associative, 5-HT, posttetanic potentiation, spontaneous release, spike broadeningl

Synaptic plasticity is a fundamental means by which environmental cues alter the function of the nervous system in both development and learning. Of particular interest are associative forms of synaptic plasticity - changes in synaptic strength triggered by the nearly simultaneous occurrence of two cellular stimuli. These types of plasticity seem excellent candidates for cellular mechanisms underlying selective synapse stabilization (Collingridge and Singer, 1990; Cline, 1991) and associative learning (Byrne, 1987; Abrams and Kandel, 1988; Hawkins et al., 1993). A number of associative forms of synaptic plasticity have been described, all of which require neuronal activity as at least one of the associative cellular stimuli, including activitydependent enhancement of presynaptic facilitation (Hawkins et al., 1983; Walters and Byrne, 1983) and inhibition (Small et al., 1989), long-term potentiation (Levy and Steward, 1979; Barrionuevo and Brown, 1983; Walters and Byrne, 1985), and longterm depression (Ito et al., 1982; Stanton and Sejnowski, 1989).

One of these associative and activity-dependent forms of plasticity has heen identified in cellular studies of classical conditioning in the marine mollusk Aplysia californica: activity-dependent enhancement of heterosynaptic facilitation at siphon sensory neuron synapses, the first afferent relay in the gill and siphon withdrawal circuit (Hawkins, 1991). Pairing a touch to the siphon, which triggers reflexive withdrawal, with aversive shock to the animal's tail produces a conditioned enhancement of the withdrawal response (Carew et al., 1981, 1983). By itself, tail shock activates modulatory interneurons that heterosynaptically facilitate transmitter release from sensory neuron terminals (Hawkins et al., 1981; Hawkins and Schacher, 1989; Mackey et al., 1989). When sensory neuron activity similar to that produced by siphon touch is paired with and immediately precedes this neuromodulatory input, the magnitude and duration of this facilitation arc cnhanced (Hawkins et al., 1983; Walters and Byrne, 1983; Buonomano and Byrne, 1990).

Pairing-specific facilitation is thought to involve an augmentation of the mechanisms underlying heterosynaptic facilitation alone. Three different transmitters contribute to the presynaptic facilitation induced by tail shock: $5-\mathrm{HT}$, small cardioactive peptide (SCP), and the as yet unidentified transmitter released by 
the L29 group of modulatory interneurons (Brunelli et al., 1976; Hawkins et al., 1981; Abrams et al., 1984; Glanzman et al., 1989; Mackey et al., 1989; Mercer et al., 1991). The most extensively studied of these is 5-HT, which facilitates release from sensory neurons by two different processes. The first involves closure of $\mathrm{K}^{+}$channels, which broadens the presynaptic action potential, increasing $\mathrm{Ca}^{2+}$ influx at transmitter release sites (Klein et al., 1982; Siegelbaum et al., 1982; Baxter and Byrne, 1990; Hochner and Kandel, 1992; Eliot et al., 1993). This effect is mediated in part by cAMP-dependent protein kinase (Castellucci et al., 1982; Shuster et al., 1985; Goldsmith and Abrams, 1992). The second process is independent of changes in the action potential and involves either vesicle mobilization or a direct enhancement of exocytosis (Hochner et al., 1986b). This second process is mediated, at least in part, by protein kinase $\mathrm{C}$, and is reflected by an increased frequency of spontaneous transmitter release in cultured sensory-motor neuron synapses (Braha et al., 1990; Dale and Kandel, 1990; Ghirardi et al., 1992).

How does paired activity enhance facilitation by $5-\mathrm{HT}$ ? Hawkins et al. (1983) found that spike broadening is increased in a pairing-specific manner, and Abrams (1985) demonstrated that this associative effect requires $\mathrm{Ca}^{2+}$ influx during pairing. These findings suggested that $\mathrm{Ca}^{3+}$ influx during the paired activity enhances the first process of 5-HT facilitation, cAMP-dependent action potential broadening. Biochemical studies support this model, indicating that $\mathrm{Ca}^{2+}$ augments cAMP production by stimulating adenylate cyclase in concert with 5-HT (Ocorr et al., 1985; Eliot et al., 1989; Abrams et al., 1991; Yovell and Abrams, 1992). However, several questions remain concerning the molecular mechanism of the associative facilitation. For example, previous findings do not rule out a role for the second process in pairing-specific facilitation. Because the second process is mediated in large part by protein kinase $\mathrm{C}$, which is also dually activated by 5-HT and intracellular $\mathrm{Ca}^{2+}$ (Sacktor and Schwartz, 1990; Sossin and Schwartz, 1992), this mobilizationlike process seems a good candidate for pairing-specific enhancement. Also, if pairing-specific facilitation requires $\mathrm{Ca}^{2+}$ influx, what is the source of this influx? Aplysia sensory neurons are known to have at least two types of voltage-dependent $\mathrm{Ca}^{2+}$ channels, a dihydropyridine-sensitive, noninactivating channel similar to the vertebrate L-type, and a more rapidly inactivating. dihydropyridine-insensitive channel (Edmonds et al., 1990). Although L-type channels are present in regions containing synaptic terminals, they play no role in synaptic transmission or its facilitation by 5-HT (Edmonds et al., 1990; Eliot et al., 1993). However, the L-current may be an essential source of $\mathrm{Ca}^{2+}$ influx underlying pairing-specific facilitation, particularly because it is itself substantially enhanced by 5-HT (Braha et al., 1993).

The ability to reconstitute synapses between individual Aplysia sensory and motor neurons in culture has proven advantageous for analyzing the mechanisms underlying various forms of synaptic plasticity. Cultured sensory-motor neuron synapses have been found to retain nearly every nonassociative form of plasticity they exhibit in vivo, including short-term homosynaptic depression and potentiation, and both short- and long-term heterosynaptic facilitation and inhibition (Montarolo et al., 1986, 1988; Rayport and Schacher, 1986; Schacher et al., 1990; Eliot et al., 1990). Can associative forms of plasticity also be reproduced in culture? We undertook a study of associative activitydependent facilitation in culture to determine whether the plasticity is inherent to the synapse, and if so, to exploit this simple system in further studying its underlying mechanisms. These studies in turn addressed several questions: (1) Is the associative facilitation presynaptic? (2) Does it involve an enhancement of the second or spike-independent process of facilitation? (3) Does it require protein kinase $C$ activation? (4) Does it involve an enhancement of the first process in facilitation (spike broadening) and enhanced production of cAMP? (5) Does it require $\mathrm{Ca}^{2+}$ influx through L-type $\mathrm{Ca}^{2+}$ channels?

\section{Materials and Methods}

Cell culture. Synapses were reconstituted in vitro between Aplysia pleural mechanosensory neurons and one of two classes of motor neurons: either the gill motor neuron, L7, obtained from juvenile ( $1-5 \mathrm{gm})$ animals, or LFS siphon motor neurons, obtained from adult ( $70-120 \mathrm{gm})$ animals. LFS motor neurons were isolated based on their size, pigmentation, and location in the left ventral abdominal ganglion, mostly caudal and a bit lateral to the LE cluster. However, it is likely that a few of the follower neurons were not LFS type, because up to 12 cells were found in a single ganglion onto which sensory ncurons madc sizablc synaptic conncctions in culture, whereas only seven or eight such cells have been found actually to cause siphon movements (Hickie and Walters, 1990). Although the identity of the follower neurons is thus somewhat ambiguous, they will be referred to as LFS cells, because it is likely that at least half were properly of this class. All of these cells had similar properties: 50 $100 \mu \mathrm{m}$ size, unpigmented appearance, two or three major axon branches, and a characteristic "notch" in their membrane potential when released from very hyperpolarized levels $(-80$ to $-90 \mathrm{mV})$, probably due to A-type $\mathrm{K}^{+}$current activation.

Cells were dissociated and cultured as described by Rayport and Schacher (1986). Briefly, abdominal and pleural ganglia were incubated at $34^{\circ} \mathrm{C}$ for $2.5 \mathrm{hr}$ in $1 \%$ protease (type IX, Sigma) dissolved in L15 medium (Flow Laboratories) supplemented to achieve the following final concentrations: $385 \mathrm{~mm} \mathrm{NaCl}, 10 \mathrm{~mm} \mathrm{KCl}, 11 \mathrm{~mm} \mathrm{CaCl}_{2}, 28 \mathrm{~mm}$ $\mathrm{MgCl}_{2}, 27 \mathrm{~mm} \mathrm{MgSO}_{4}, 2.3 \mathrm{~mm} \mathrm{NaHCO}, 35 \mathrm{~mm}$ D-glucose. Ganglia were desheathed and then individual cells removed with a long, flexibletipped glass electrode. Pleural sensory neurons were plated in contact with a motor neuron in a $50 \mathrm{~mm}$ poly-L-lysine-coated plastic culture dish containing $2.5 \mathrm{ml}$ of culture medium ( $50 \%$ supplemented $\mathrm{L} 15+$ $50 \%$ sterile filtered hemolymph). For cultures containing two sensory neurons synapsing on a common motor neuron (Fig. $1 A$ ), the sensory neurons were plated as far apart from each other on the target motor neuron as possible to minimize electrical coupling between them (Bank and Schacher, 1992). Synaptic connections appeared as early as $24 \mathrm{hr}$ after plating and rest at room temperature. Dishes were transferred to an $18^{\circ} \mathrm{C}$ incubator after $24 \mathrm{hr}$.

Synaptic electrophysiology. Experiments were performed on cultures 3-5 d after plating ( $2-4 \mathrm{~d}$ for spontaneous release experiments) in medium containing $50 \%$ supplemented $\mathrm{L} 15$ and $50 \%$ artificial seawater ( $460 \mathrm{~mm} \mathrm{NaCl}, 10 \mathrm{~mm} \mathrm{KCl}, 11 \mathrm{~mm} \mathrm{CaCl}_{2}, 55 \mathrm{~mm} \mathrm{MgCl}, 2 \mathrm{~mm} \mathrm{NaHCO}$, $10 \mathrm{~mm}$ Na-HEPES, $\mathrm{pH}$ 7.6). All experiments except those measuring spontaneous transmitter release were conducted at $15-18^{\circ} \mathrm{C}$. Motor neurons were impaled with sharp microelectrodes (15-25 M $\Omega$ ) filled with $2 \mathrm{~m} \mathrm{~K}$-acetate, $0.5 \mathrm{~m} \mathrm{KCl}$, buffered to $\mathrm{pH} 7.2$ with $10 \mathrm{~mm} \mathrm{~K}$-HEPES and held at -80 to $-90 \mathrm{mV}$ when recording evoked EPSPs using the bridge circuit on an Axoclamp 2A amplifier (Axon Instruments, Burlingame CA). Sensory neurons were stimulated extracellularly, using unpolished patch pipettes (1-3 M $\Omega$ ) filled with extracellular solution. At each trial, the intensity of a $0.1 \mathrm{msec}$ current pulse to the sensory neuron was slowly increased until a single EPSP appeared in the motor neuron; tetanus was elicited by raising the intensity $20 \%$ above threshold level of the last trial. Although such a procedure would result in failures if the sensory neuron had been stimulated intracellularly, with extracellular stimulation it reliably produced a single action potential in the sensory neuron for every pulse in the train, as judged by the occurrence of EPSPs recorded in the motor neuron at high gain. The few experiments in which failures were observed were discarded. Each synapse was tested once shortly after motor neuron impalement, and then rested $15 \mathrm{~min}$ before beginning the experiment. In experiments using two presynaptic neurons, both sensory neurons were impaled at the end of the experiment to test the degree of electrical coupling between them. The experiment was discarded if tetanic stimulation of one sensory 
neuron delivered in the presence of 5-HT produced any action potentials in the other sensory neuron.

Measurements of spontaneous release. Spontaneous release was measured exclusively in cultures containing a single sensory neuron in contact with a single motor neuron as described previously (Dale and Kandel, 1990). We used two different methods to record miniature postsynaptic responses in pairing experiments. Initially, we recorded mEPSPs in L7 motor neurons under current clamp using sharp electrodes. In later experiments, we switched to whole cell voltage clamp and recorded mEPSCs in the smaller LFS motor neurons using patch pipettes (four of nine paired, and four of nine unpaired experiments). For current-clamp experiments, L7 was impaled with a beveled, lowresistance microelectrode (6-8 M 2 ). Voltage-clamp experiments were performed in the whole cell configuration (Hamill et al., 1981) using 3$4 \mathrm{M} \Omega$ patch pipettes filled with $465 \mathrm{mM}$ Cs-gluconate, $2 \mathrm{~mm} \mathrm{MgCl}_{2}, 1$

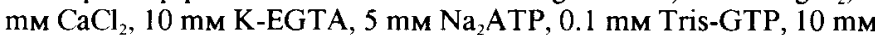
reduced glutathione, and $50 \mathrm{~mm}$ Cs-HEPES, buffered to $\mathrm{pH} 7.2$ with $\mathrm{CsOH}$. Although mEPSCs were recorded using continuous single electrode voltage clamp on the Axoclamp, we switched back to current clamp to measure evoked EPSPs in these experiments.

Measurements of mEPSP and mEPSC frequencies and amplitudes were performed as described by Dale and Kandel (1990). Postsynaptic recordings were made at both high gain (AC coupled) to measure miniature EPSPs or EPSCs and low gain (DC coupled) to measure evoked EPSPs. High-gain recordings were filtered (at $300 \mathrm{~Hz}$ for mEPSPs and $2-4 \mathrm{kHz}$ for mEPSCs), digitized (4 kHz sampling frequency), and analyzed using a PDP-11 computer and programs written in Basic-23. The mEPSPs or mEPSCs were detected manually based on fast rise time and amplitude greater than peak-to-peak noise level (see Fig. 6). Peak amplitude and the interval between minis were then determined automatically. The better signal-to-noise of mEPSCs measured under whole-cell voltage clamp made it possible to use automatic thresholding to detect them. Histograms of mini amplitudes were fit with 1-3 gaussian distributions determined using the simplex algorithm and the method of maximum likelihood (Dale and Kandel, 1990). In most L 7 recordings, peak-to-peak noise was about $40 \mu \mathrm{V}$, so this was used as a lower cutoff for mEPSP amplitude. Average unitary mEPSP amplitude was $76.3 \pm$ $6.6(+\mathrm{SEM}) \mu \mathrm{V}(n=9)$. For LFS recordings, the noise cutoff varied more between experiments but was generally set at $6 \mathrm{pA}$. Average unitary mEPSC amplitude was $17.5 \pm 3.3 \mathrm{pA}(n=7)$. Distributions of both $\mathrm{mEPSP}$ and $\mathrm{mEPSC}$ sizes showed distinct peaks, generally in multiple intervals of the first amplitude, suggesting that the lower-end cutoff does not distort the first peak. Noise levels often rose during experiments using either type of recording. Experiments were rejected when the noise exceeded the smallest signals detectable at the beginning of the experiment. Because of the difficulty of maintaining low-noise recordings for an extended period of time, the effects of paired or unpaired training on spontaneous release were compared between different synapses alternately given either paired or unpaired training.

Drug application. Modulatory transmitters (5-HT or SCP) were applied as a $5 \mu \mathrm{l}$ bolus in the immediate vicinity of the cells, either using a Hamilton syringe as previously described (Schacher et al., 1990) or, for temporal pairing experiments, using a $5 \mu \mathrm{l}$ capillary glass pipette positioned just upstream and aimed at the cells. In the latter case, timing was controlled by a Picospritzer (General Valve). The $5 \mu$ l of extracellular medium contained either $50 \mu \mathrm{M} 5-\mathrm{HT}$ or $50 \mu \mathrm{M} \mathrm{SCPb}$ (Peninsula Laboratories). A constant bath perfusion (approximately $1 \mathrm{ml} / \mathrm{min}$ ) controlled washout from the $2.5 \mathrm{ml}$ culture dish. In some experiments, $0.5 \%$ fast green was also included to monitor the location of the puff. Fast green was no longer visible near the cells $30 \mathrm{sec}$ after the puff. In some later experiments (see Figs. 7, 9), 5-HT was applied by microperfusing a $10 \mu \mathrm{M}$ solution immediately over the cells beginning at the onset of the tetanus, and then switching to control saline microperfusion after $15 \mathrm{sec}$. Although it is difficult to determine the exact duration of 5-HT exposure, it appeared comparable for both application methods, based on another measure of 5-HT action in sensory neurons, reduced spike-frequency accommodation (Klein et al., 1986). Thus, in parallel experiments, 5-HT produced large increases in the number of sensory neuron action potentials elicited by fixed intracellular depolarizing step commands ("anti-accommodation"), and this effect was completely reversed in 60-90 sec for both methods of application (not shown). Since this duration can be estimated with greater certainty than 5-HT washout itself, we have used it in the figures (see Figs. 4, 5, 7, 9, 13) to indicate 5-IIT exposure. Thus, the time indicated by the 5-IIT bar $(90 \mathrm{sec})$ is an upper estimate of the maximum duration of 5-HT exposure in synaptic experiments.
The protein kinase inhibitor $\mathrm{H} 7$ (Seikagaku) was dissolved in water ( $10 \mathrm{~mm}$ stock solution) and applied by microperfusion in a final concentration of 200 or $400 \mu \mathrm{M}$ in extracellular solution. Nitrendipine (gift of A. Scriabine, Miles Pharmaceutical) was made fresh daily as a 10 $\mathrm{mM}$ stock in dimethyl sulfoxide (DMSO) and bath applied in a final concentration of 2 or $10 \mu \mathrm{M}$.

Isolated sensory clusters. Clusters of pleural mechanosensory neurons were dissected out, treated with $0.5-1.0 \%$ protease (type XIV, Sigma) in artificial seawater at $18^{\circ} \mathrm{C}$ for $30-90 \mathrm{~min}$, and allowed to recover for $2-6 \mathrm{~d}$ at $18^{\circ} \mathrm{C}$ in artificial seawater supplemented with amino acids, vitamins, sugar, penicillin, and streptomycin (Eisenstadt et al., 1973). The pleural sensory neuron cluster is a relatively pure preparation containing only sensory neuron cell bodies and neuropil (Walters et al., 1983). The protease treatment is needed to disrupt synaptic transmission from any terminals of neuromodulatory cells that survive following isolation.

Clusters were pinned to the Sylgard floor of a $40 \mu \mathrm{l}$ well continuously perfused at $40 \mu \mathrm{l} / \mathrm{sec}$ with supplemented artificial seawater (without amino acids) containing $100 \mu \mathrm{M}$ 3-isobutyl-1-methylxanthine (IBMX) diluted from a $0.5 \mathrm{~m}$ stock solution in DMSO. IBMX was added to block cAMP breakdown, thereby focusing our measurements on effects due to changes in cAMP production. A single sensory neuron was impaled with a 10-20 M electrode containing $2.5 \mathrm{M} \mathrm{KCl}$. The cell was allowed to rest for $10 \mathrm{~min}$ following impalement before an experiment was begun. During training, the entire population of sensory neurons was stimulated by delivering a $20 \mathrm{~Hz}, 2 \mathrm{sec}$ train of $1 \mathrm{msec}, 100 \mathrm{~V}$ biphasic shocks ("tetanus") between two silver wires positioned on opposite sides of the well (see Fig. 10). Cells in the cluster fire action potentials one-for-one with the shocks. 5 -HT ( 4 or $10 \mu \mathrm{M}$, with $0.01 \%$ fast green) was applied for $30 \mathrm{sec}$ beginning $0.5 \mathrm{sec}$ (paired) or $1 \mathrm{~min}$ (unpaired) after the beginning of the tetanus. Timing of the 5-HT delivery was controlled by an electronic valve in the perfusion line. The 5 -HT concentration reached steady state within a few seconds during washin and washout. Sensory neuron excitability was quantified as the number of action potentials resulting from a $500 \mathrm{msec}$ depolarizing intracellular current pulse whose magnitude was adjusted before training to produce one action potential. Action potential duration was measured from the peak to the point on the falling phase that was at $30 \%$ of peak amplitude, using a laboratory interface to an IBM-compatible PC and commercially available software (SPIKE, Hilal Associates, Englewood, $\mathrm{NJ}$ ). The action potential was measured at the end of a $20 \mathrm{~Hz}, 1 \mathrm{sec}$ train, which inactivates the delayed rectifier $\mathrm{K}^{+}$current, making the action potential duration more sensitive to changes in $\mathrm{S}$-type $\mathrm{K}^{+}$current.

Data analysis. Data in the text and figures are presented as mean \pm SEM, and except for Figure 11 are normalized to pretest magnitude (EPSP amplitude or action potential duration). The results shown in Figure 3 are corrected for homosynaptic depression by dividing normalized experimental values by the average normalized control (test alone) amplitude at each time point. In experiments measuring spontaneous transmitter release (see Fig. 7), data were $\log$ transformed and then normalized, so measurements of both evoked EPSP amplitude and mini frequency are presented as geometric mean \pm SEM. Normalized data were analyzed by two-way ANOVA with repeated measures. When a significant main effect of treatment or interaction was found, the time course of the effect was then examined using either one-way ANOVA at each time point followed by Dunnett's two-tailed $t$ test for multiple groups, or using $t$ tests at each individual time point when only two groups were being compared.

\section{Results}

Pairing 5-HT or SCP with tetanic stimulation prolongs facilitation compared to either procedure alone

Aplysia sensory-motor neuron synapses reconstituted in culture exhibit both homosynaptic facilitation in response to tetanic stimulation and heterosynaptic facilitation in response to neuromodulator application (Rayport and Schacher, 1986; Schacher et al., 1990; Eliot et al., 1990). As a first step in assessing whether associative facilitation also occurs in culture, we compared the effects of each treatment alone with their effect when delivered in combination. Two groups of cultures were used for these experiments, each containing two sensory neurons synapsing onto a single L7 motor neuron. In the first group, one 


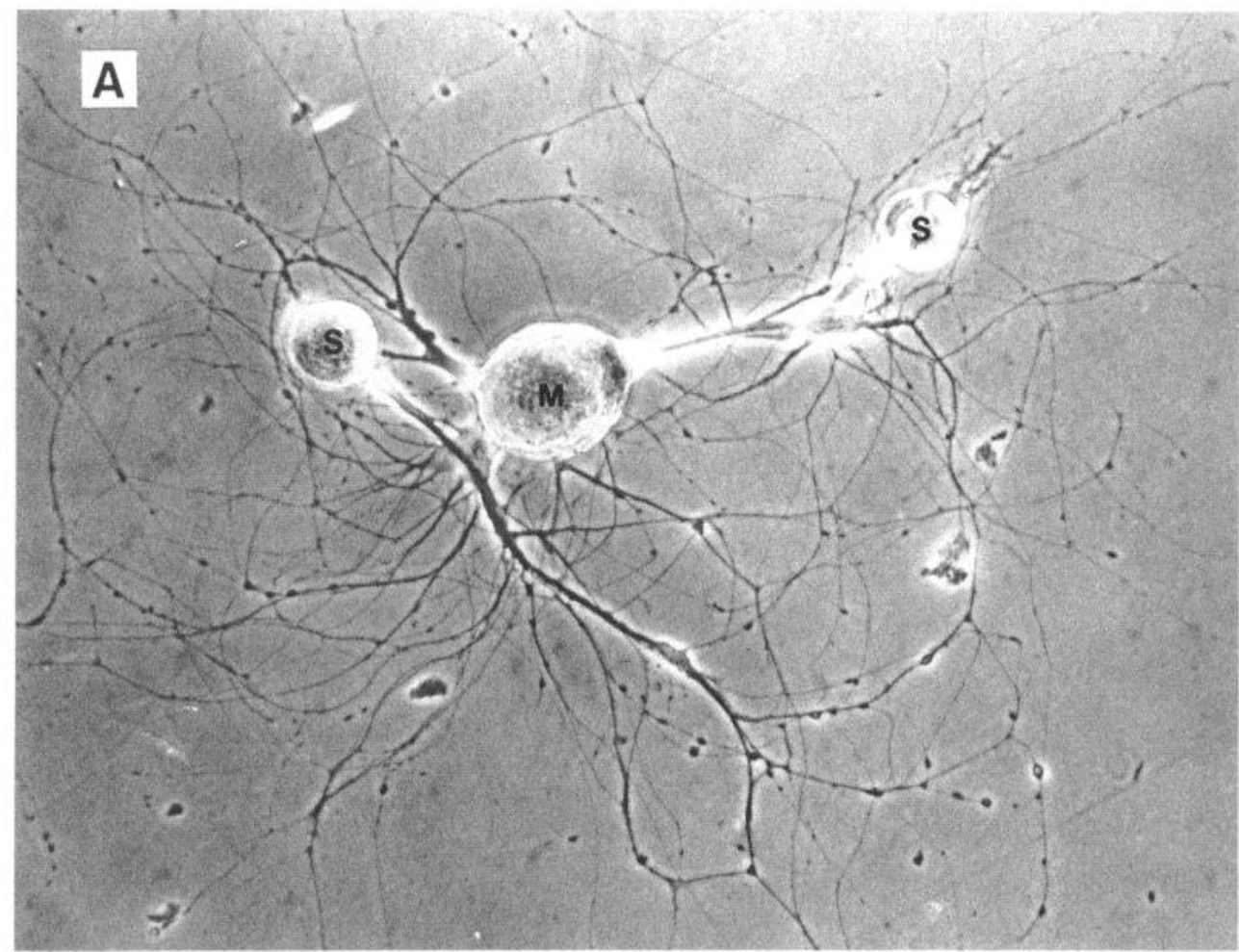

$\mathrm{B}_{1}$

CONTROL SN

TET. ALONE SN

$\mathrm{B}_{\mathbf{2}}$

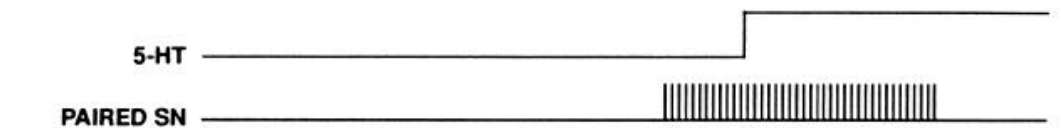

5-HT ALONE SN

$\mathrm{C}_{1}$

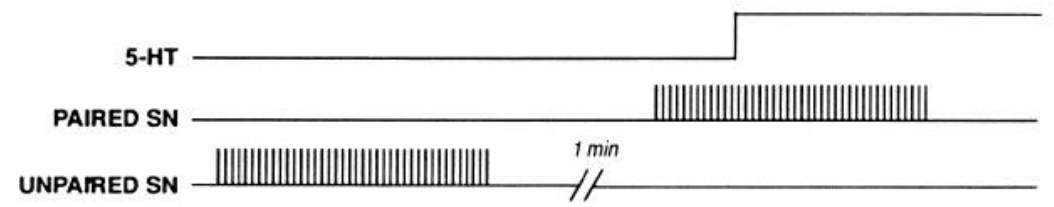

$\mathrm{C}_{2}$

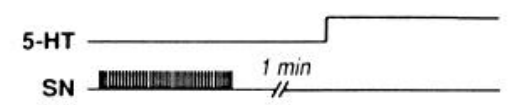

UNPAIRED
REST

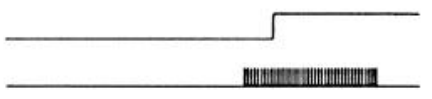

PAIRED
Figure 1. Experimental design. A, Sensory to motor neuron synapses were established in culture using either one or two presynaptic mechanosensory neurons $(S)$ plated in contact with a single motor neuron $(M)$, in this case a siphon motor neuron from the LFS group. $B$, Design of experiments comparing tetanus alone, 5 -HT alone, paired training, and no training (control). Two groups of cultures containing two sensory neurons $(S N)$ synapsing on a single L7 motor neuron were used. In one group $\left(B_{1}\right)$ one sensory neuron was tetanized $(20 \mathrm{~Hz}, 2 \mathrm{sec})$, while the other received no training, to serve as a control for homosynaptic depression. In the other group $\left(B_{2}\right)$, one sensory neuron was tetanized immediately before application of 5-HT or SCP (and thus received paired training), while the other received no stimulation, to measure the effect of transmitter alone. Each synapse was tested once, $10 \mathrm{~min}$ before training, and then posttested four times as indicated in Figures 2 and 3. $C$, Design of experiments comparing paired and unpaired training. Two groups of cultures containing a single LFS motor neuron and either one or two presynaptic sensory neurons were used. In the two sensory neuron design $\left(C_{1}\right)$, one sensory neuron received tetanus paired with a $5 \mu \mathrm{l}$ puff of $50 \mu \mathrm{M} 5$-HT $(0.5 \mathrm{sec}$ after onset of tetanus), while the other sensory neuron received tetanus $1 \mathrm{~min}$ before the $5-\mathrm{HT}$. In the one sensory neuron design $\left(C_{2}\right)$, a single presynaptic sensory neuron received either paired or unpaired training in round $\mathrm{A}$, followed by the opposite form of training in round B. Each synapse was pretested twice, at $5 \mathrm{~min}$ intervals, and then tested four times after training, as indicated in Results. The rest period was $30 \mathrm{~min}$ for synaptic experiments and $15 \mathrm{~min}$ for experiments measuring action potential width in pleural sensory neuron clusters. sensory neuron served as control for the effects of normal synaptic depression while the other sensory neuron received a tetanus to assess the magnitude and duration of posttetanic potentiation (PTP; Fig. $1 B_{l}$ ). Similarly, in the second group only one sensory neuron was tetanized, but in these cultures a brief application of 5-HT was delivered immediately after the onset of the tetanus, so that one synapse was exposed to 5-HT alone while the other received 5-HT paired with tetanus (Fig. $1 B_{2}$ ). In another study, we found that PTP does not occur in culture when the presynaptic sensory neuron is impaled with an intracellular electrode (Eliot, 1991), so sensory neurons were stimulated extracellularly, as described in Materials and Methods. 

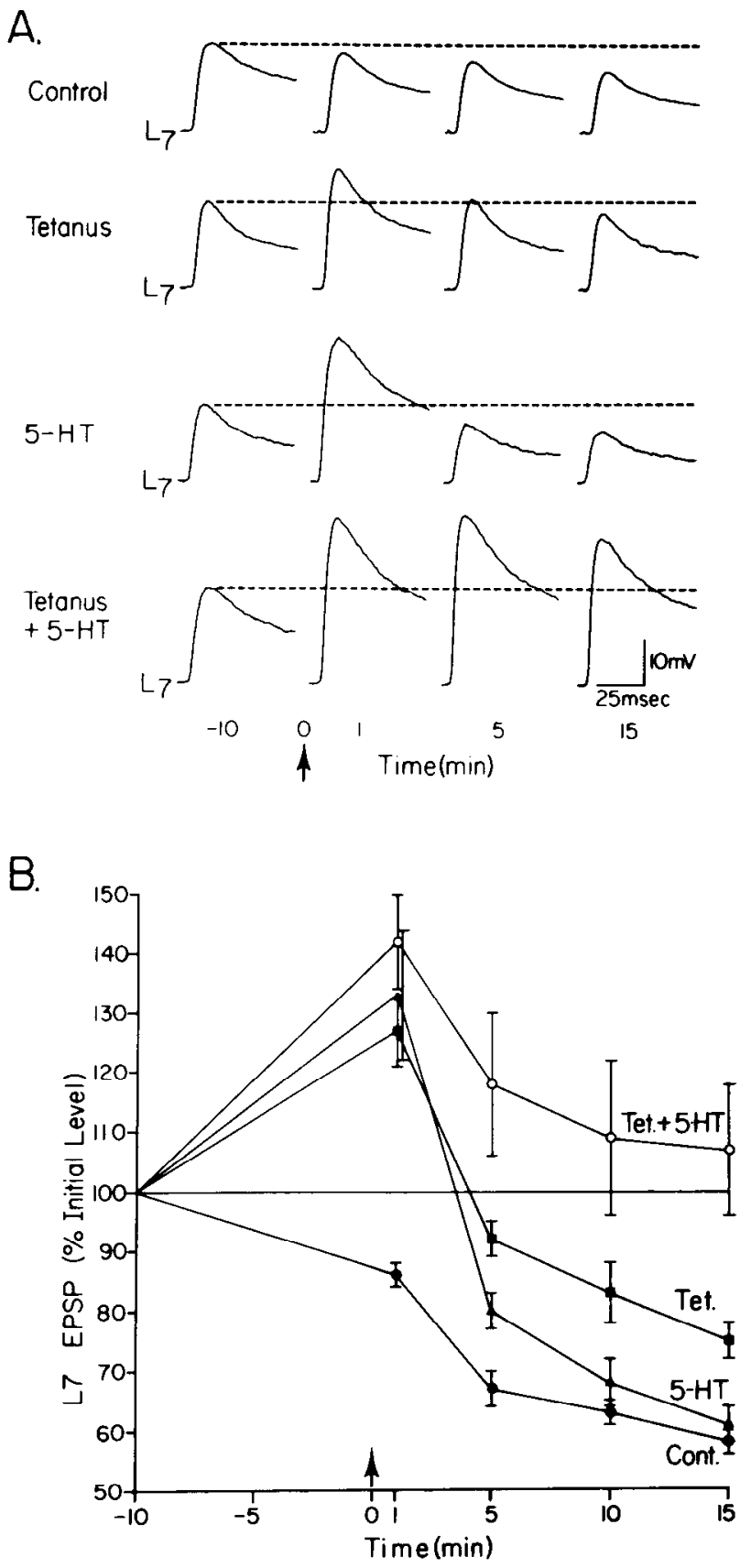

Figure 2. Pairing 5-HT with tetanus prolongs facilitation compared to either treatment alone. $A$, EPSPs recorded in $\mathrm{L} 7$ motor neurons in response to extracellular stimulation of presynaptic sensory neurons. Four different treatments were delivered (at arrow) to two separate groups of cultures, as illustrated in Figure $1, B$, and $B$, In the first group of cultures, the sensory neuron receiving test alone undergoes homosynaptic depression, while the sensory neuron receiving tetanus alone exhibits PTP lasting 5-10 min. In the second group, the sensory neuron exposed to 5-HT alone is facilitated only briefly after transmitter application, while the sensory neuron receiving tetanus paired with 5-HT remains facilitated up to $15 \mathrm{~min}$ after training. $B$, Group data. Cultures containing two sensory neurons synapsing on a common L7 motor neuron were trained in two groups ( $n=10$ in each): test alone (Cont.) versus PTP (Tet.), and 5-HT versus paired $($ Tet $.+5-H T)$. Each synapse was pretested once, received its treatment $10 \mathrm{~min}$ later (indicated by arrow), and then posttested 1, 5, 10, and 15 min after treatment. Error bars indicate SEM. Initial EPSP amplitudes were $12.7 \pm 1.8 \mathrm{mV}$ (Cont.), $13.4 \pm 2.2(5-H T), 13.6 \pm 1.6($ Tet.$)$, and $14.1 \pm 2.6($ Tet $.+5-H T)$, and were not significantly different by one-way ANOVA.

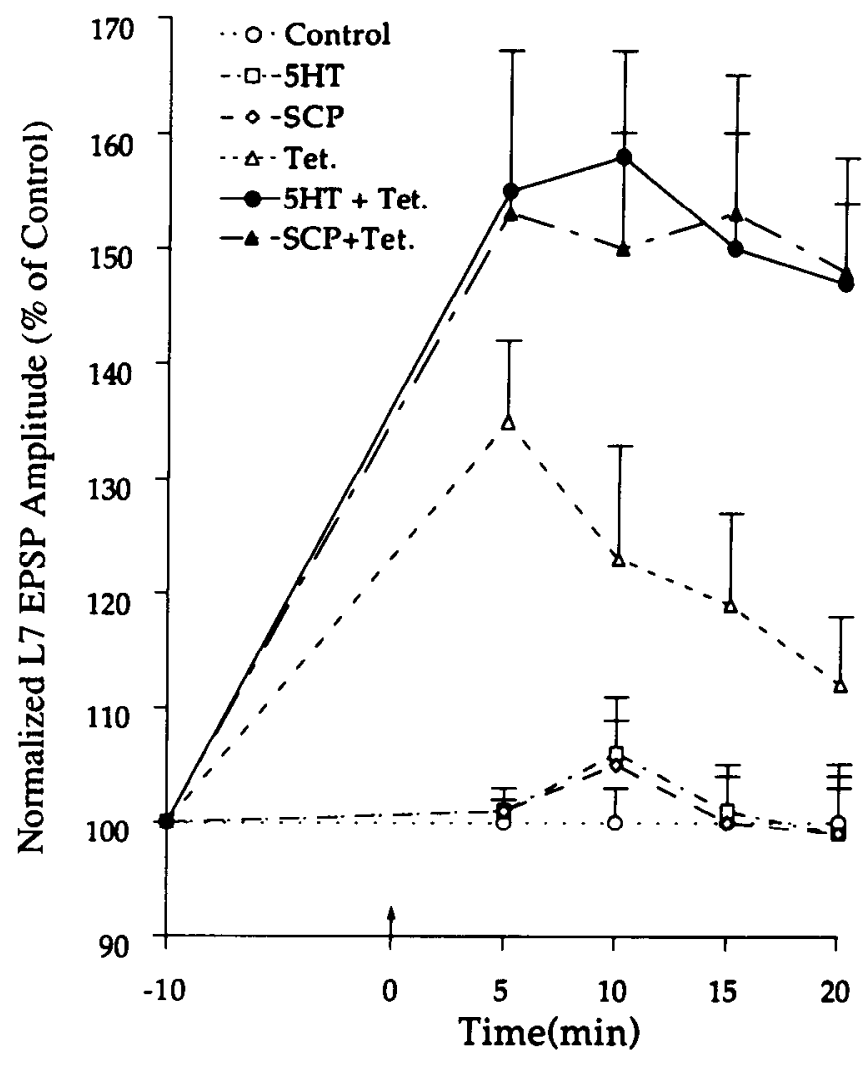

Figure 3. Either 5-HT or SCP can induce the paired enhancement of facilitation, which appears nondecremental when normalized for homosynaptic depression. Results are from three groups of cultures, using the experimental design of Figure $1 B:(1)$ test alone (Control) versus tetanus alone $(n=5),(2) 5$-HT alone versus 5 -HT paired with tetanus $(n=6)$, and (3) SCP alone versus SCP paired with tetanus $(n=6)$. Synapses were pretested $10 \mathrm{~min}$ before treatment (at arrow), and then posttested $5,10,15$, and $20 \mathrm{~min}$ after treatment. Responses were normalized to pretest level within individual experiments (results comparable to Fig. 2 ), and then normalized again to the average amplitude of the control group at each time point, to evaluate the time course of each treatment independently of homosynaptic depression. Initial EPSP amplitudes were $11.3 \pm 2.3$ (Control), $16.0 \pm 4.4(5 H T), 12.5 \pm 2.4(S C P), 12.0$ $\pm 2.6($ Tet. $), 14.8 \pm 1.8(5 H T+$ Tet. $)$, and $10.8 \pm 1.5(S C P+T e t$.$) ,$ and were not significantly different by one-way ANOVA.

Results from both groups of cultures are plotted together in Figure 2. Each of the three experimental treatments facilitated the synapse compared to control, but the treatments differed in the duration of facilitation (overall, $F_{3,36}=12.52$ for treatment and $F_{12.144}=7.96$ for treatment $\times$ time, $p<0.001$; treatment also produced a significant effect at every posttest, $p<0.001$ by one-way ANOVA). 5-HT alone produces facilitation that is evident 1 min after application ( $131 \pm 8 \%$, Dunnett's two-tailed $t=4.79, p<0.01$ compared to control), but with continuous bath perfusion the facilitation is no longer significant $5 \mathrm{~min}$ after application. Tetanic stimulation alone produces a comparable amount of initial facilitation $(126 \pm 7 \%, t=3.80, p<0.01)$ and the effect lasts somewhat longer (at $5 \mathrm{~min}, t=2.53, p<$ 0.05 ). Nevertheless, the PTP is clearly decremental, such that 10 min after tetanization the synapse is no longer significantly enhanced $(t=1.96, \mathrm{NS})$. In contrast to these short-lived effects, pairing 5-HT with the same tetanic stimulation produces considerably longer-lasting facilitation, remaining enhanced $15 \mathrm{~min}$ after training $(t=5.73, p<0.01)$.

As is evident from the decay of the control group responses 
A

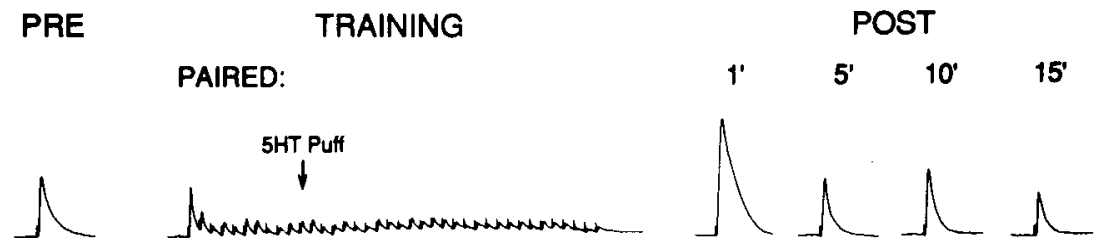

UNPAIRED: TETANUS 1' BEFORE 5HT

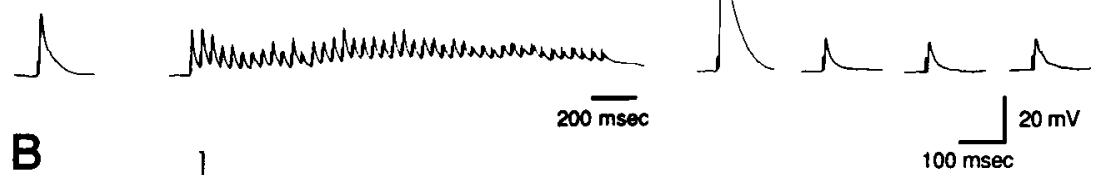

B

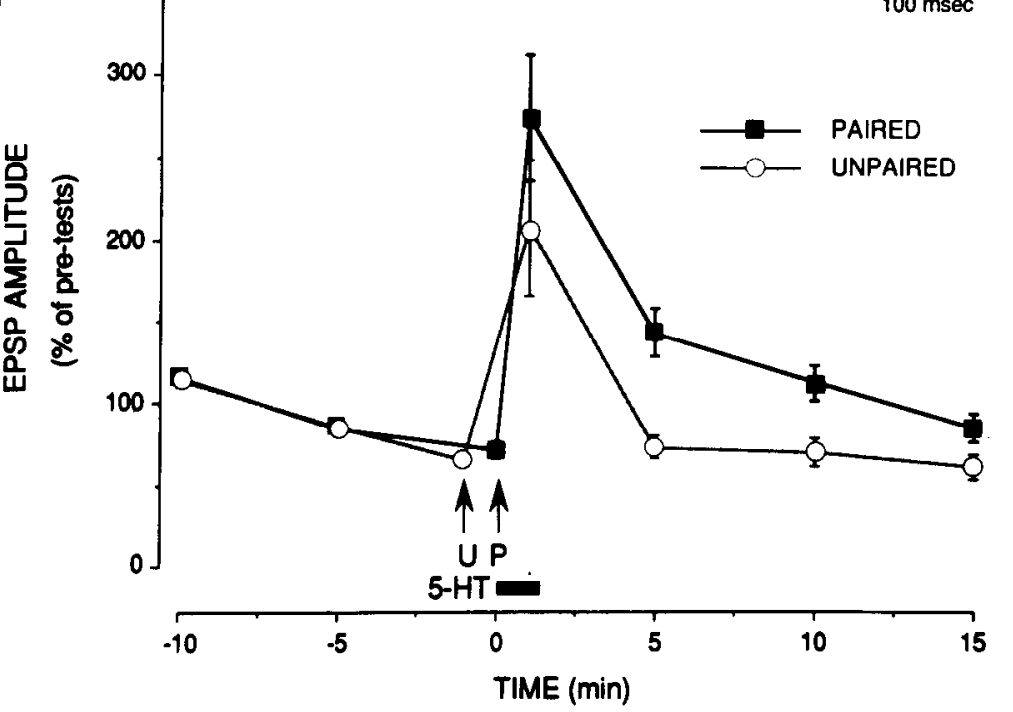

Figure 4. Associative facilitation using a two sensory neuron design. $A$, EPSPs recorded in an LFS motor neuron in response to separate extracellular stimulation of two presynaptic sensory neurons using the protocol in Figure $\mathrm{I} C_{I}$ Each synapse was pretested twice at 5 min intervals (the second pretest of each synapse is shown here) and then one synapse received tetanus paired with 5-HT application while the other synapse was tetanized $1 \mathrm{~min}$ before $5-\mathrm{HT}$. Posttests reveal initial facilitation of both synapses, but only the paired synapse remains facilitated at later time points. $B$, Group data from 14 experiments. Overall, pairing significantly enhanced facilitation. The horizontal bar next to 5-HT in this and subsequent figures indicates the onset and outer limit of washout following the puff at $t$ $=0 \mathrm{~min}$ (see Materials and Methods). Arrows indicate delivery of extracellular tetanus to the unpaired $(U)$ and paired $(P)$ sensory neurons. Average EPSP amplitude before training was $15.0 \pm 2.8 \mathrm{mV}$ for the paired synapses and $17.7 \pm 3.3 \mathrm{mV}$ for the unpaired synapscs.
(Fig. 2), sensory neuron synapses exhibit profound depression, even with very low-frequency stimulation. To minimize this effect, we performed another set of experiments in which we eliminated the test 1 min after training. We also added a third group of cultures to this experiment, to test whether facilitation by another modulatory transmitter, SCP, is similarly enhanced by paired activity. The results with $5-\mathrm{HT}$, tetanus, $5-\mathrm{HT}+$ tetanus, and control were similar to those shown in Figure 2. To evaluate the facilitatory effects independently of homosynaptic depression, we normalized EPSP amplitudes by the average amplitude of the "test alone" group at each time point. These data are displayed in Figure 3.

These experiments reveal that either 5-HT or SCP when paired with tetanus produce considerably longer-lasting facilitation than transmitter or tetanus treatment alone. The paired enhancement is virtually indistinguishable for the two transmitters. Although the facilitatory effect of either transmitter alone washes out before the first posttest, the paired group remains substantially facilitated up to $20 \mathrm{~min}$ later. Moreover, this facilitation appears nondecremental, especially compared to the facilitation by tetanus alone. Facilitation was significant overall $\left(F_{3,2 \gamma}=14.30\right.$ for treatment and $F_{4.112}=6.91$ for treatment $\times$ time, $p<0.001$ ) and at each posttest (by one-way ANOVA, $p<0.001$ ). As in the previous experiment, the tetanus alone group is significantly facilitated for $5 \mathrm{~min}$ after training (Dunnett's $t=3.15, p<0.05$ ) and then decays back toward control $(t=2.29$ at $10 \mathrm{~min}$, NS). In contrast, both paired groups are significantly facilitated at every posttest (Dunnett's $t$ test, $p<0.01$ ). Significant interactions in the analysis of variance reveal that pairing tetanus with either SCP or 5-HT enhances facilitation more than would be expected by simply adding PTP and heterosynaptic facilitation (overall, $F_{1,10}=8.46$ for tetanus $\times 5$-HT interaction, $p<0.02$; $F_{1,9}=4.25$ for tetanus $\times$ SCP interaction, $p<0.05$, one-tailed). For both transmitters, this interaction influences the duration, more than the initial magnitude of facilitation, since it is significant at 15 and $20 \mathrm{~min}$, but not at 5 and $10 \mathrm{~min}$ posttraining ( $p<0.05$ and $p<0.01$ at 15 and $20 \mathrm{~min}$, respectively, for pairing with either transmitter, one-way ANOVA).

These experiments demonstrate that pairing either facilitatory transmitter, SCP or 5-HT, with tetanic stimulation, produces a long-lasting synaptic enhancement. In contrast to the brief facilitation induced by a short transmitter exposure, and the somewhat longer-lasting, but still decremental PTP induced by tetanus alone, the facilitation induced by pairing is nondecremental for at least $20 \mathrm{~min}$ when corrected for homosynaptic depression.

Long-lasting facilitation requires the temporal pairing of 5-HT and tetanus

The synergistic facilitation induced by pairing transmitter with tetanus suggests that this form of plasticity depends on temporal 
A

ROUND A: UNPAIRED TRAINING
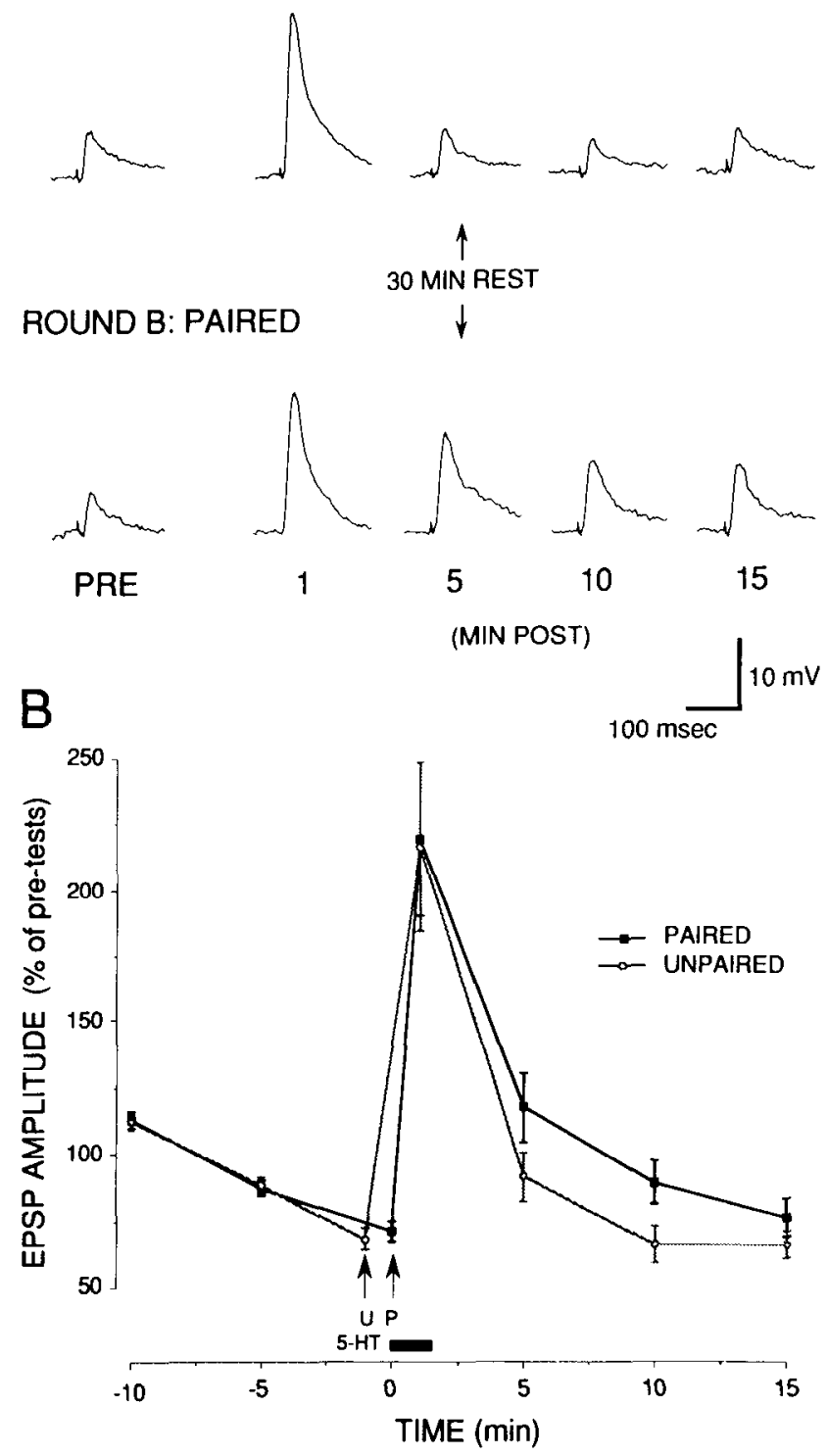

Figure 5. Associative facilitation using a one sensory neuron design. $A$, Pairing-specific facilitation in a synapse given unpaired training in the first round, followed by paired training in the second round, using the protocol in Figure $1 C$, As in the two cell experiments, pairing increased the duration but not the initial amplitude of facilitation. $B$, Group data from 20 experiments counterbalanced for order of training. Excluding the 1 min postlest, pairing produced an overall enhancement of facilitation. Average EPSP amplitude was $17.3 \pm 2.5 \mathrm{mV}$ before paired training and $16.4 \pm 2.4 \mathrm{mV}$ before unpaired training.

conjunction of the two stimuli, like the facilitation induced by pairing presynaptic activity with facilitatory input in the ganglion (Hawkins et al., 1983; Walters and Byrne, 1983). However, it is possible that transmitters and tetanus interactively facilitate the synapse without requiring temporal conjunction. To test whether this facilitation requires temporal pairing, we thus compared paired training to training in which the two stimuli are delivered unpaired in time.

We performed two sets of experiments to assess the temporal specificity of activity-dependent facilitation in culture. The first experimental design used cultures containing two sensory neurons synapsing on a common siphon motor neuron (Fig. $1 A, C_{l}$ ). Lach synapse was pretested twice, at $5 \mathrm{~min}$ intervals, and then a coin flip determined which would be the paired cell. Pairing consisted of a tetanus followed $0.5 \mathrm{sec}$ after onset by a $5 \mu \mathrm{l}$ puff of $50 \mu \mathrm{M} \mathrm{5-HT}$. The unpaired cell received the tetanus $1 \mathrm{~min}$ prior to 5-HT application.

Figure $4 A$ illustrates the effects of paired and unpaired training in one experiment. Both paired and unpaired cells show substantial facilitation 1 min after training. However, the paired synapse remains enhanced many minutes after training, whereas the unpaired synapse does not. Group data are presented in Figure $4 B$. Overall, pairing produced a significant enhancement of facilitation compared to unpaired training $\left(F_{1.13}=7.17, p\right.$ $<0.02$ ). Individual $t$ tests show the difference between paired and unpaired groups is not significant at the 1 min posttest, but is significant at 5,10 , and 15 min posttests $(p<0.01,0.01$, and 0.02 , respectively).

In the sccond set of experiments, an $\mathrm{A} / \mathrm{B}$ design was used to assess the effects of pairing within a single synapse (Fig. $1 C_{2}$ ). A single sensory neuron received either paired or unpaired training in a first round, was rested $30 \mathrm{~min}$, and then received the alternate form of training in a second round. Figure 5 shows the results of these experiments. Again, paired training produced significantly more facilitation than unpaired training. There was no difference between paired and unpaired groups $1 \mathrm{~min}$ after training, but from 5 to 15 min posttraining, the paired group was significantly more facilitated than the unpaired group $\left(F_{1,19}\right.$ $=5.49, p<0.05$ ). Individual $t$ tests indicate the paired group was significantly more facilitated than the unpaired group at each of the 5, 10, and $15 \mathrm{~min}$ posttests ( $p<0.05$, one-tailed).

In both sets of experiments, we aligned the time of posttests to the time of 5-HT application to eliminate the contribution of 5-HT washout to the duration of facilitation. As a consequence, the unpaired synapse was posttested $1 \mathrm{~min}$ later, relative to its tetanus, than the paired cell. This difference probably does not account for the observed pairing effect, however, because in later experiments we aligned the posttests relative to the tetanus - that is, the unpaired cell was tested 1 min earlier than the paired cell-and the pairing effect persisted (data not shown).

These two sets of experiments demonstrate that the persistent facilitation requires the temporal pairing of 5-HT and tetanus. Pairing appeared not to affect the initial magnitude of facilitation, although this could be a ceiling effect. The paired effect was more robust using the two-sensory neuron design than the $\mathrm{A} / \mathrm{B}$ design. This difference may be due to the long-lasting nature of the facilitation, although we did not find any systematic order effect in the $\mathrm{A} / \mathrm{B}$ experiments.

\section{Analysis of spontaneous release during pairing-specific facilitation}

The ability to study pairing-specific facilitation in culture provides a number of advantages for mechanistic analysis. We exploited the ability to measure spontaneous transmitter release-mEPSPs or mEPSCs - that unambiguously reflect the release process of a single presynaptic neuron in cultures containing just one sensory and one motor neuron. Measurements of mini amplitude provide an assay for changes in quantal amplitude that could implicate a postsynaptic mechanism of pairing-specific facilitation. Measurements of mini frequency allowed us to test whether pairing-specific facilitation involves 
A PAIRED

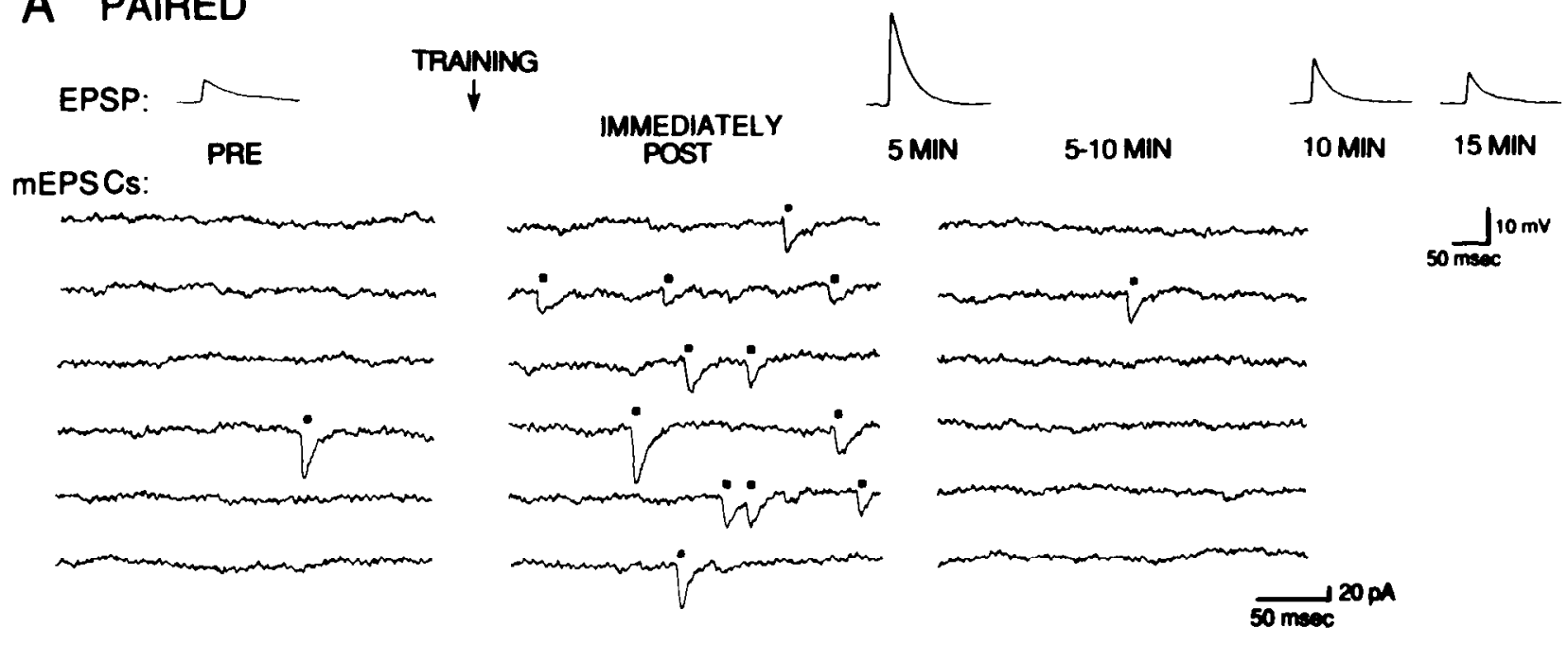

B UNPAIRED

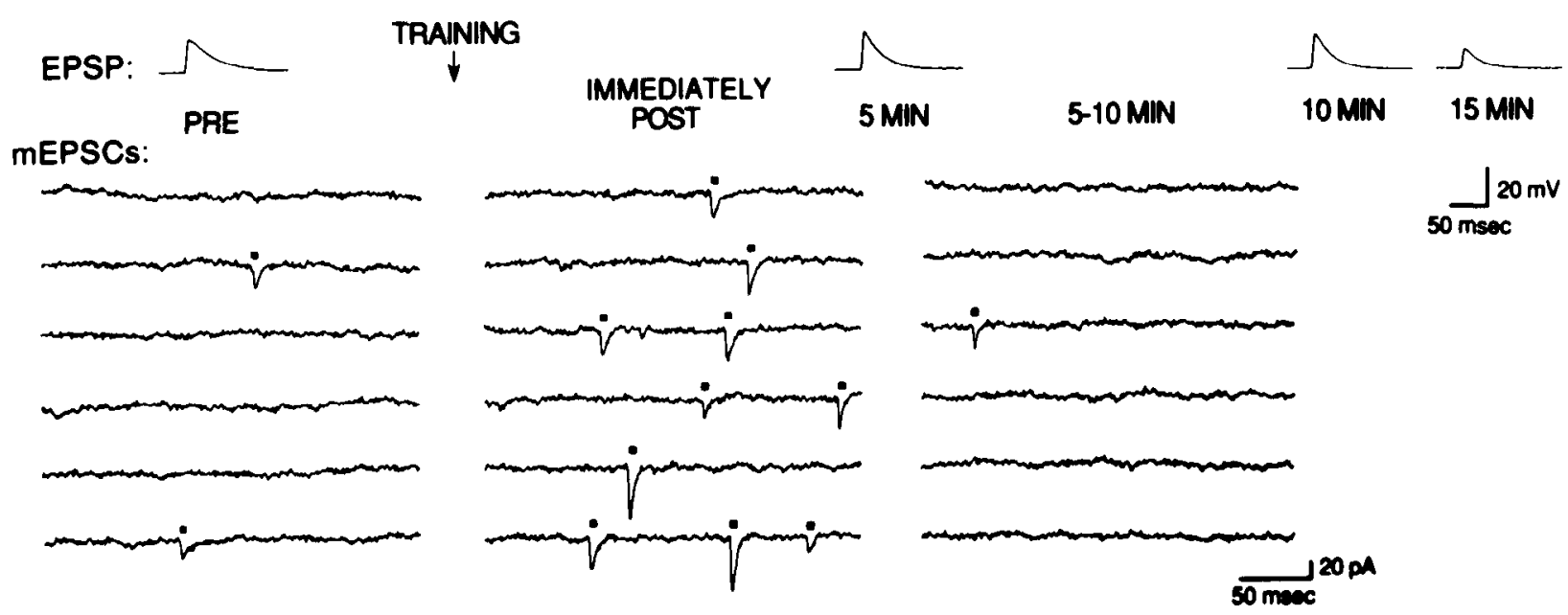

Figure 6. Fffect of paired and unpaired training on evoked and spontaneous release in cultures containing a single sensory neuron synapsing on a single LFS motor neuron. Each synapse was given only one form of training, paired $(A)$ or unpaired $(B)$, in alternate experiments. mEPSCs (indicated by dots) were recorded under whole-cell voltage clamp $(-50 \mathrm{mV}$ holding potential) and evoked EPSPs were recorded in bridge mode, with the motor neuron hyperpolarized to $-90 \mathrm{mV}$. Both paired and unpaired training produce large initial increases in mini frequency, but the enhancement is not pairing specific and does not persist as long as pairing-specific facilitation of evoked release. The actual frequency of spontaneous release is considerably lower in these synapses than is depicted. Sweeps containing mEPSCs were selected to illustrate their amplitude, kinetics, and relative changes in frequency after training.

presynaptic modulation of processes independent of the action potential or excitation-secretion coupling.

Figure 6 illustrates the effects of paired and unpaired training on evoked and spontaneous release in two different LFS cultures. Resting mEPSC frequency is quite low $\left(0.080+0.011 \mathrm{sec}^{-1}\right.$ in paired cultures, and $0.078 \pm 0.021 \mathrm{sec}^{-1}$ in unpaired cultures, $n=9$ each). Following both paired and unpaired training, the frequency rises dramatically. This initial increase decays fairly rapidly, such that 5-10 min after training, both paired and unpaired mini frequencies remain only slightly elevated above resting levels. In contrast to the comparable decay of paired and unpaired mEPSC frequency, the evoked EPSP remains facilitated up to $15 \mathrm{~min}$ after paired training. Because the previous set of experiments indicated that there is no pairing effect $1 \mathrm{~min}$ after training, we omitted the 1 min posttest of the evoked EPSP in these experiments to provide an uninterrupted interval for measuring spontaneous release after either paired or unpaired training.

Overall, pairing produced a significant enhancement of evoked EPSP facilitation $\left(F_{1,12}=8.49, p<0.02\right.$, three-way ANOVA with motor neuron type as an additional factor; see Materials and Methods), but had no significant effect on spontaneous release frequency $\left(F_{1,12}=0.54\right.$, NS, three-way ANOVA; Fig. 7). Although mini frequency was significantly elevated for both groups together $(p<0.01$ at all intervals up to $5 \mathrm{~min}$ posttest, Dunnett's multiple range test), mini frequencies of paired and unpaired groups were not significantly different from each other at any time point. The time course of facilitation of the unpaired 
A

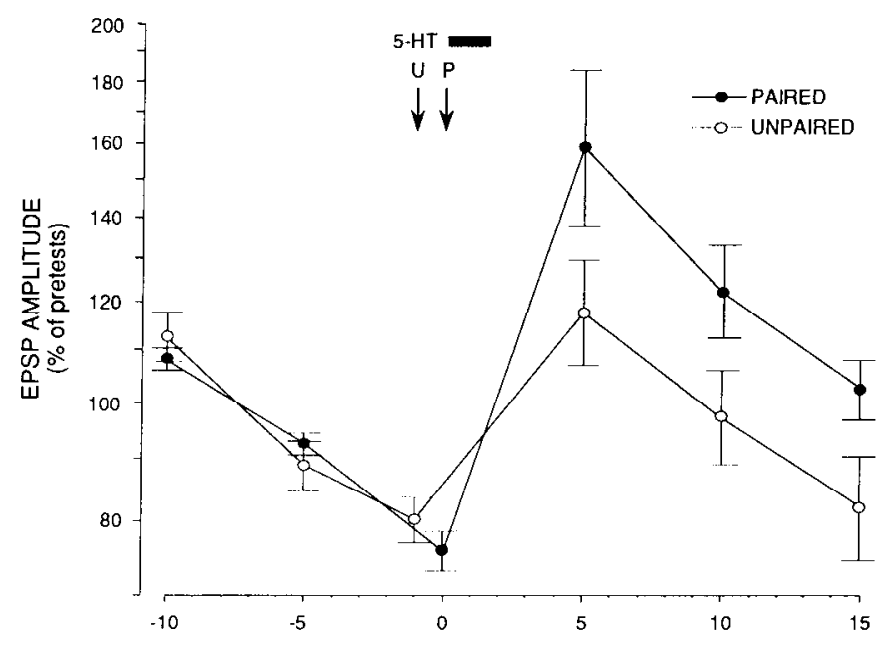

B

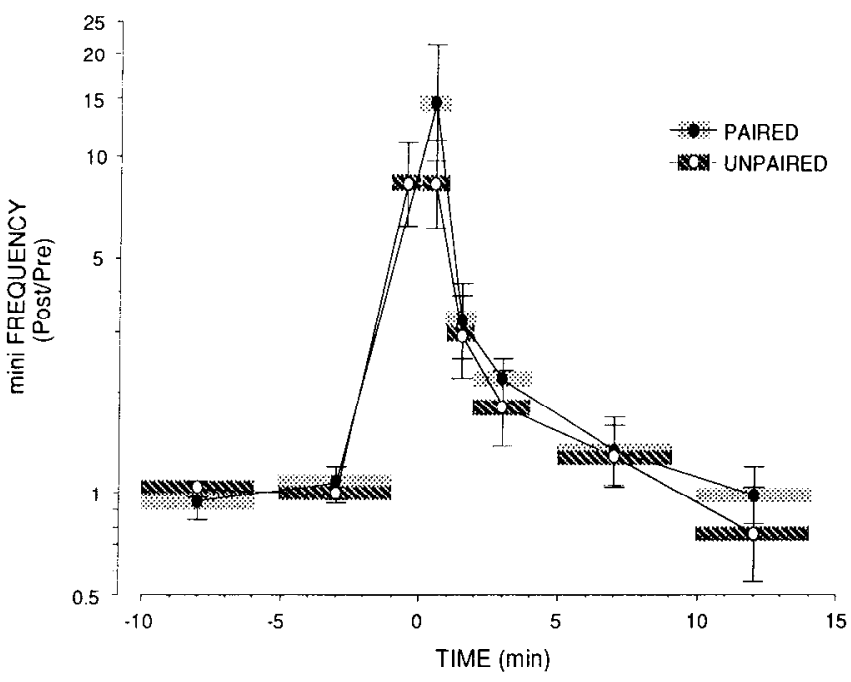

Figure 7. Pairing-specific facilitation is not accompanied by an increase in spontaneous release frequency. $A$, Group data showing pairingspecific facilitation of evoked release ( $n=8$ each for paired and unpaired groups; training regiment was alternated between experiments). The data are plotted on a log scale so as to be comparable to spontaneous release data. Average EPSP amplitude before training was $22.7 \pm 5.1 \mathrm{mV}$ for the paired synapses and $19.5 \pm 1.9 \mathrm{mV}$ for the unpaired synapses. $B$, Spontaneous release was not enhanced in a pairing-specific manner ( $n$ = 9; one paired and one unpaired experiment were not included in $A$ because their EPSPs produced spikes in the postsynaptic neuron). Although release frequency is substantially elevated immediately after training, it does not remain elevated for the duration of facilitation. In these experiments, 5-HT was applied by microperfusion, as described in Materials and Methods.

evoked EPSP was similar to the time course of spontaneous release frequency. Thus, the unpaired group was significantly facilitated relative to pretest amplitude only at $5 \mathrm{~min}$ posttraining ( $p<0.01$, Dunnett's multiple range test). In contrast, the paired EPSP remained significantly facilitated at each of the 5 , 10 , and $15 \mathrm{~min}$ posttests $(p<0.01$ at 5 and $10 \mathrm{~min}, p<0.05$ at $15 \mathrm{~min}$ ). While spontaneous release in the paired group tended to be slightly higher than in the unpaired group 10-15 min after training, an analysis of covariance indicates that the effect of pairing on the evoked EPSP at that time was significantly greater
A

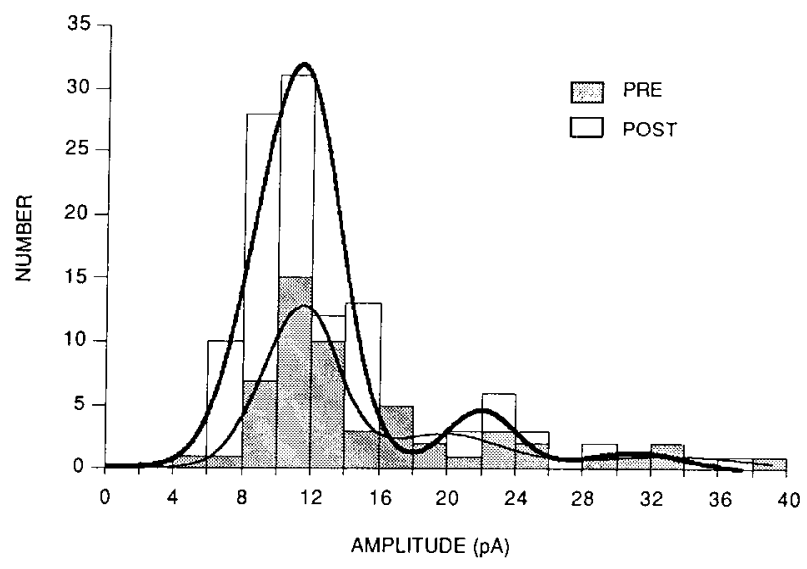

$\mathrm{B}$

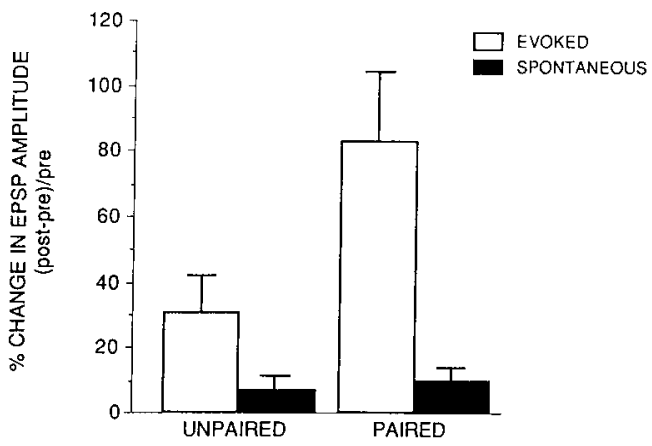

Figure 8. Pairing-specific facilitation is not accounted for by a change in quantal size. $A$, Distribution of mEPSC amplitudes before (from -10 to $-1 \mathrm{~min}$ ) and after (from 0 to $+15 \mathrm{~min}$ ) paired training in one experiment from the group depicted in Figure 7. Both distributions could be fit with three gaussian distributions. The mean and SDs of the peaks before training were $11.2 \pm 2.2,19.8 \pm 3.4$, and $33.1 \pm 4.6 \mathrm{pA}(n=$ $55)$, and after training were $11.0 \pm 2.5,22.2 \pm 2.1$, and $31.5 \pm 3.1 \mathrm{pA}$ $(n=118)$. The mean amplitude of the apparent unitary mEPSCs is thus unchanged by paired training, although the evoked EPSP in this experiment was facilitated to $196 \%$ of the amplitude of the first EPSP of the tetanus. $B$, Average facilitation of the evoked EPSP at 5, 10, and 15 min posttests (normalized to the first EPSP of the tetanus) is significant for both paired and unpaired groups $(t=8.35$ for paired, $p<$ $0.05 ; t=11.42$ for unpaired, $p<0.01 ; n=8$ in each group), whereas quantal size is not significantly changed in either group $(t=1.36$ for paired, $t=0.71$ for unpaired, NS). Moreover, paired facilitation of the evoked EPSP is greater than unpaired facilitation $(t=2.15, p<0.05)$, whereas there is no difference between changes in paired and unpaired quantal size $(t=0.43$, NS). Unitary mini amplitude was determined for each experiment as in $A$, by fitting Gaussian curves to the amplitude distribution of spontaneous events in all the intervals before or after training, excluding 1 min windows during which the evoked EPSP was tested.

than would be expected from the difference in spontaneous release frequency $\left(F_{1,11}=6.29, p<0.05\right)$.

Analysis of unitary mEPSP and mEPSC amplitudes before and after training indicates that changes in quantal size also cannot account for pairing-specific facilitation. Amplitude distributions of mEPSPs or mEPSCs generally yielded multiple gaussian peaks, with the vast majority of minis fitting in the first, or lowest, amplitude class (Fig. 8A). The mean amplitude of larger events tended to be integral multiples of the smallest mean amplitude, although the small number of such events makes a quantitative comparison impossible. We thus com- 
pared the mean amplitude of the smallest or presumed unitary events before and after the two different training procedures. Averaging all posttests for the paired group, the evoked EPSP increased by $79.0 \pm 21.4 \%$ (normalized to first EPSP of the tetanus), while the unitary amplitudes showed a small increase of $6.0 \pm 4.4 \%$ during the $15 \mathrm{~min}$ after training. Similarly, in the unpaired group, the evoked EPSP increased by $27.0 \pm 11.1 \%$, whercas unitary size increased by $3.3 \pm 4.6 \%$ (Fig. $8 B$ ). These small changes in unitary size are not statistically significant, nor is there any difference between paired and unpaired groups. These results suggest that changes in quantal size cannot account for pairing-specific facilitation.

The finding that quantal size is unaltered indicates that pairing-specific facilitation is mediated presynaptically, by an increase in the number of quanta released. That the paired enhancement of evoked release is not mirrored by an increased frequency of spontaneous release furthermore suggests that whatever the presynaptic mechanism underlying the associative effect, it involves processes unique to evoked release.

\section{Pairing-specific facilitation is not blocked by $\mathrm{H7}$}

5-HT by itself is known to increase spontaneous transmitter release frequency (Dale and Kandel, 1990), an effect that is mediated by protein kinase C (Ghirardi et al., 1992). The fact that pairing activity with $5-\mathrm{HT}$ does not augment this increase in spontaneous release suggests that protein kinase $C$ does not contribute significantly to pairing-specific facilitation. This conclusion is further supported by our finding that the $\mathrm{C}$ kinase inhibitor $\mathrm{H} 7$ does not block pairing-specific facilitation.

Pairing experiments were performed using cultures containing two sensory neurons (Fig. $1 C_{1}$ ). In five experiments, $200 \mu \mathbf{M ~ H 7}$ was applied $10 \mathrm{~min}$ before pretesting and remained present throughout the experiment, while in two other experiments, 400 $\mu \mathrm{M} \mathrm{H} 7$ was applied beginning 2 min before the unpaired tetanus until just after the 1 min posttest. These concentrations of $\mathrm{H} 7$ have previously been shown to block effectively the increase in sensory neuron mini frequency induced by 5-HT or phorbol esters (Braha et al., 1990; Ghirardi et al., 1992). Pooled results of these experiments are plotted in Figure 9. Consistent with its reduction of basal synaptic transmission (Eliot, 1991), H7 reduced the overall level of facilitation in both paired and unpaired groups compared to control experiments (Fig. 4). Nonetheless, pairing-specific facilitation still occurs in the presence of $\mathrm{H} 7\left(F_{1,16}=4.33\right.$ for 5-15 min posttests, $p<0.05$, one-tailed).

Thus, although protein kinase $C$ would seem to make a good candidate for a site of convergence between $\mathrm{Ca}^{2+}$ influx and 5-HT activation, it does not appear to be necessary for pairingspecific facilitation. These results are consistent with the lack of a pairing effect on spontaneous release and further support the conclusion that the second or spike-independent process of facilitation by $5-\mathrm{HT}$ is not involved in pairing-specific facilitation.

\section{Pairing 5-HT and tetanus enhances sensory neuron excitability and action potential width, consistent with enhanced production of $C A M P$}

Previous studies of associative facilitation in the ganglion indicated that it is mediated by an activity-dependent enhancement of presynaptic action potential broadening, or the first process of heterosynaptic facilitation (Hawkins et al., 1983; Abrams, 1985), likely due to an activity-dependent enhancement of cAMP production by adenylate cyclase (Ocorr et al.,

\section{PAIRING - SPECIFIC FACILITATION IN H7}

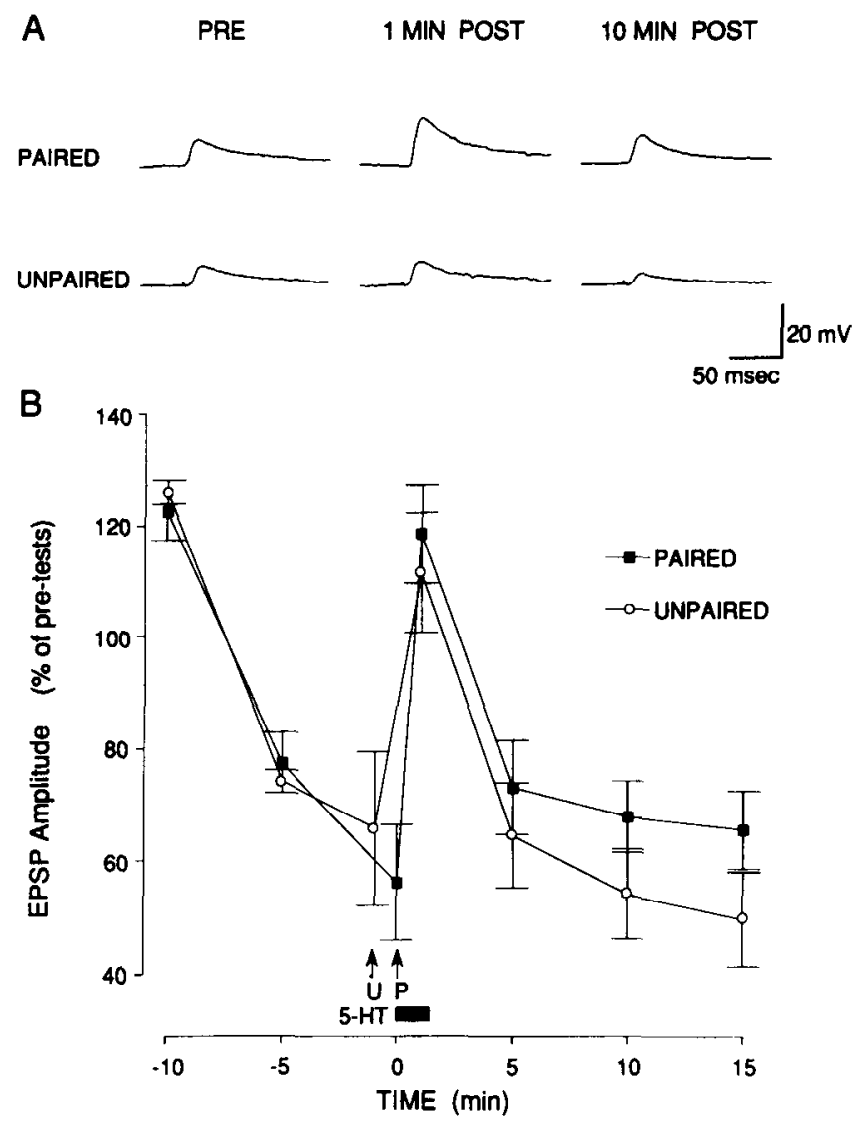

Figure 9. The $\mathrm{C}$ kinase inhibitor $\mathrm{H} 7$ does not block pairing-specific facilitation. A, Example of pairing-specific facilitation induced despite training in the presence of $200 \mu \mathrm{M} \mathrm{H} 7$. These experiments were performed on cultures containing two sensory cells, using the design depicted in Figure $1 C_{l}, B$, Group data from seven experiments, five performed in the continuous presence of $\mathrm{H} 7$ and two performed with $\mathrm{H} 7$ present from -3 to $+1 \mathrm{~min}$. Despite the pairing effect, the overall level of facilitation was quite low, consistent with H7's inhibitory effect on synaptic transmission (Eliot, 1991). In these experiments, 5-HT was applied by microperfusion.

1985; Yovell and Abrams, 1992). The above experiments indirectly support this conclusion by providing evidence against a contribution of the protein kinase $\mathrm{C}$-mediated second process. However, it is possible that yet additional mechanisms underlie the pairing-specific facilitation in culture, particularly because we used a somewhat different training protocol than has been used in pairing experiments in the ganglion. In the ganglion, activity-dependent facilitation was generally induced using multiple trials pairing brief trains of action potentials (6-10) in the sensory neuron, with nerve shock as the modulatory input (Hawkins et al., 1983; Walters and Byrne, 1983). The more robust, one-trial protocol we have used in these culture experiments might engage an entirely different intracellular mechanism.

To test the role of the "first process" in associative facilitation with the one-trial protocol, and to develop procedures for future biochemical measurements of cAMP levels, we used isolated clusters of sensory neurons from the pleural ganglion, and stimulated them en masse as depicted in Figure 10 using the same $20 \mathrm{~Hz}, 2$ sec tetanus as in synaptic experiments. The experiments 

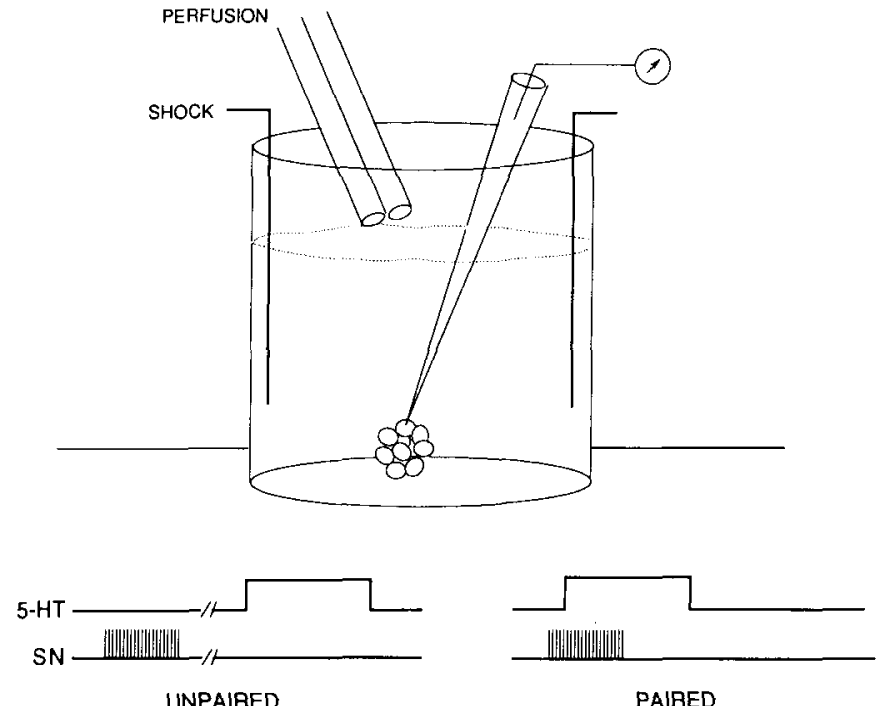

PAIRED

Figure 10. Physical arrangement for training isolated clusters of pleural sensory neurons. The pairing protocol for isolated sensory neurons was the same as synaptic experiments (Fig. 1C). A train of action potentials (tetanus) was produced in the sensory neurons by extracellular shock either $0.5 \mathrm{sec}$ (paircd) or $60 \mathrm{sec}$ (unpaired) before a $30 \mathrm{sec}$ pulse of 5-HT delivered through the perfusion. An intracellular electrode in one of the cells in the cluster was used to monitor the effects of these training procedures on the biophysical properties of the cell.

were conducted in the presence of the phosphodiesterase inhibitor IBMX (100 $\mu \mathrm{M}, 0.02 \% \mathrm{DMSO})$ to rule out confounding effects of cAMP breakdown, so that the results might more directly reflect differences in adenylate cyclase activation.

In a first set of experiments, we measured excitability in individual sensory neurons in response to either the same paired ( $0.5 \mathrm{sec}$ forward) or unpaired ( 1 min forward) training procedure used in synaptic experiments (Fig. 1C). We used both left and right pleural sensory neuron clusters from a single animal, randomly assigning one to receive paired and the other unpaired training. Sensory neurons normally exhibit strong spike accommodation during a long depolarizing step command. By itself, 5-HT has a robust anti-accommodation effect (Klein et al., 1986) which appears to be a purely cAMP-mediated phenomenon as well as a selective assay of the S-type $\mathrm{K}$ current (Klein et al., 1986; Baxter and Byrne, 1990; Hochner and Kandel, 1992).

As Figure 11 illustrates, pairing tetanus with 5-HT significantly enhances sensory neuron excitability compared to unpaired training. Pairing produces more anti-accommodation than unpaired training overall on the three trials following training $\left(F_{1,11}=6.71, p<0.05\right)$, with significant differences at each of the trials by individual $t$ tests. The effect cannot be accounted for by the different interval between the tetanus and posttests for paired and unpaired groups because the tetanus by itself slightly decreases excitability. If anything, this would tend to decrease excitability of the paired group more than the unpaired group.

This associative enhancement of sensory neuron excitability suggests that the one-trial procedure enhances cAMP production in a pairing-specific manner, consistent with carlier biochemical experiments using a high $\mathrm{K}^{+}$pairing protocol (Ocorr et al., 1985). The results also suggest that the S-type $\mathrm{K}^{+}$current is down-modulated in a pairing-specific manner. To address whether this down-modulation might contribute to the synaptic facilitation, we performed a second set of experiments on isolated sensory clusters. In these experiments, we asked whether the same training procedure produces any pairing-specific changes in action potential width that could contribute to an enhancement of transmitter release.

Figure 12 illustrates the effect of various training procedures on action potential width. To enhance the contribution of the S-type $\mathrm{K}^{+}$current, we measured action potential duration at the end of a $1 \mathrm{sec}, 20 \mathrm{~Hz}$ train, which inactivates the delayed rectifier $\mathrm{K}^{+}$current. In these experiments, we used an $\mathrm{A} / \mathrm{B}$ design in which each cluster received two training sessions with a $15 \mathrm{~min}$ interval between sessions. Some cells received training with $5-\mathrm{HT}$ alone and 5-HT paired with tetanus, and others received training with tetanus alone and no training (i.e., a 4 min "rest" between the last pretest and the first posttest). Training order was counterbalanced. As has been previously demonstrated (Klein and Kandel, 1978), 5-HT alone prolongs action potential repolarization (Fig. 12A). This is reflected in a significant overall effect of 5-HT $\left(F_{1.22}=11.39, p<0.01\right)$. When 5-HT was paired with a 2 sec tetanus, this action potential broadening was significantly enhanced compared to 5 -HT alone ( $p<0.02$ on each posttest by individual $t$ tests). By contrast, tetanic stimulation alone slightly decreased action potential duration compared to the notraining control $(p<0.05$ on the first posttest). Thus, the greater broadening produced by paired training appears to be due to an interactive effect between tetanus and 5-HT, greater than the sum of either treatment alone. This result is reflected in a significant 5-HT $\times$ tetanus interaction $\left(F_{1,22}=17.71, p<0.001\right)$.

These results agree with earlier studies demonstrating that pairing activity with 5-HT prolongs action potential duration (Hawkins et al., 1983; Abrams, 1985). It seems likely that both this pairing-specific spike broadening and the pairing-specific anti-accommodation effect are mediated by the same cAMPdependent closure of S-type $\mathrm{K}$ channels, which would be expected to contribute to facilitation of the synapse.

\section{$\mathrm{Ca}^{2+}$ influx through L-type channels is not necessary for pairing-specific facilitation}

The pairing-specific increases in action potential duration and cellular excitability are consistent with the finding that cAMP concentration is increased in sensory neurons given paired as compared to unpaired exposure to 5 - $\mathrm{HT}$ and high $\mathrm{K}^{+}$depolarization (Ocorr et al., 1985). This enhancement is likely mediated by dual activation of adenylate cyclase by $\mathrm{Ca}^{2+}$ and 5-HT (Eliot et al., 1989; Abrams et al., 1991; Yovell and Abrams, 1992). Sensory neurons contain at least two different voltage-activated $\mathrm{Ca}^{2+}$ channels (Edmonds et al., 1990), raising the issue of the source of $\mathrm{Ca}^{2+}$ influx that contributes to pairing-specific facilitation. Pairing-specific facilitation may require $\mathrm{Ca}^{2+}$ influx through one specific type of channel, both channels, or neither of the identified currents. We tested whether the L-type current plays a role in pairing-specific facilitation by repeating the differential training experiments in culture in the presence of the dihydropyridine antagonist nitrendipine. We found in previous voltage-clamp experiments that $10 \mu \mathrm{M}$ nitrendipine blocks about 90\% of L-type current in sensory neurons (Eliot et al., 1993). Unlike their action in vertebrate neurons, dihydropyridine antagonists do not require depolarization to block $\mathrm{Ca}^{2+}$ currents effectively in Aplysia neurons (Nerbonne and Gurney, 1987; Edmonds et al., 1990).

By itself, nitrendipine slightly increases sensory neuron excitability (data not shown), probably due to reduction of $I_{\mathrm{k}(\mathrm{ca})}$. 
A
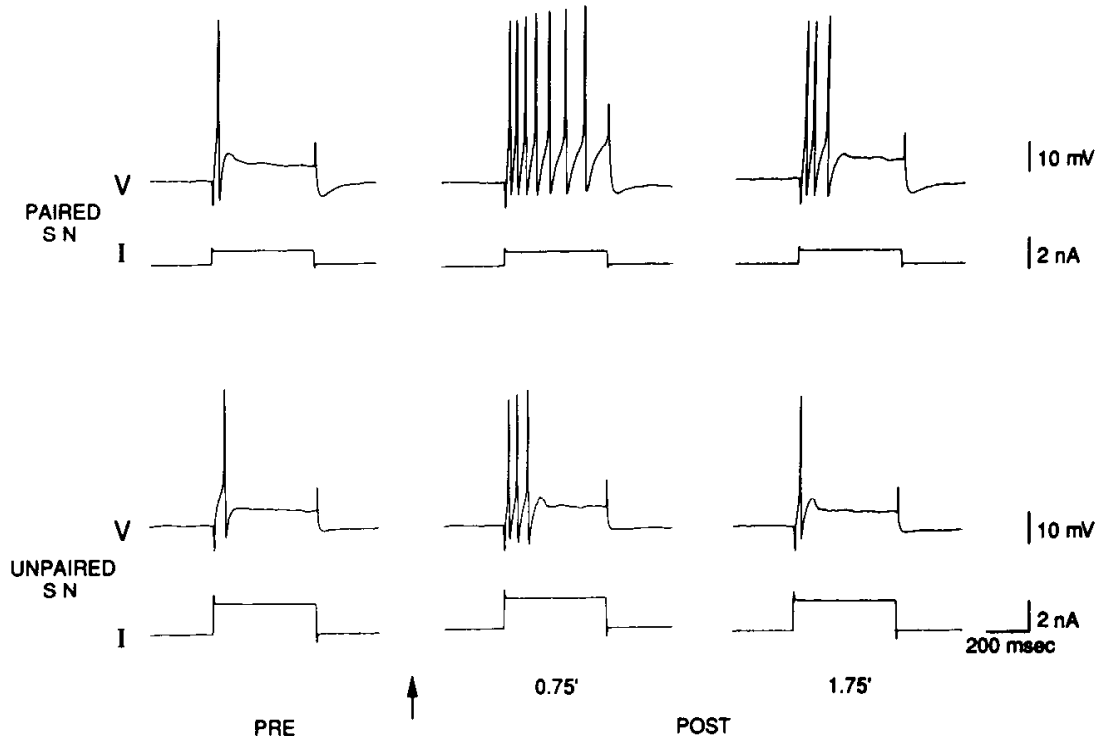

B

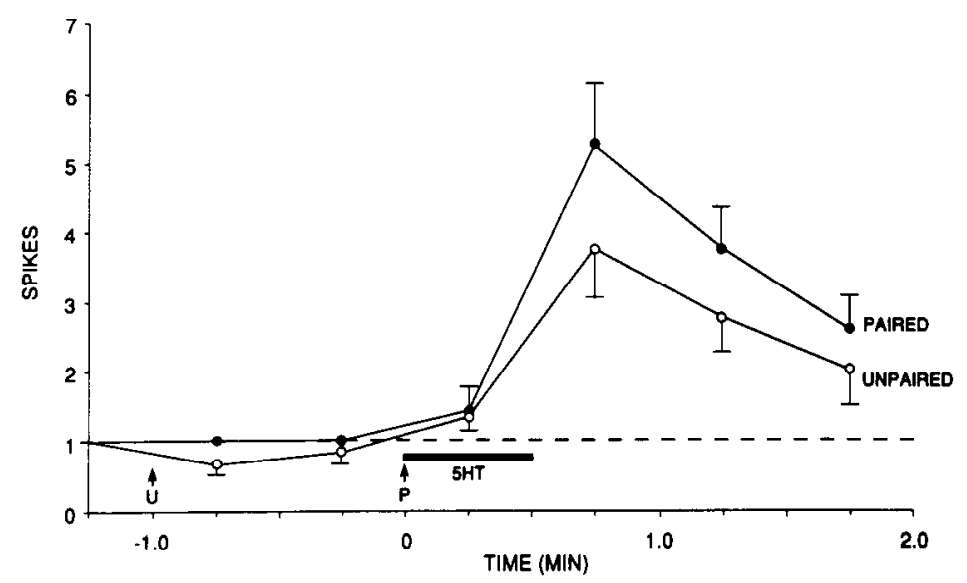

Figure 11. Pairing-specific increasc in sensory neuron excitability. $A$, An intracellular depolarizing current pulse was adjusted to produce one spike in the sensory neuron before training $(P R E)$. Paired training (top) produced a larger and longer-lasting increase in excitability or number of action potentials produced by the current pulse than unpaired training of the contralateral cluster from the same animal (bottom). $B$, Group data ( $n=12$ per group). Paired training produced a significantly greater increase in excitability than unpaired training on the three trials following training. The tetanus occurred at the arrows for paired $(P)$ and unpaired $(U)$ training, and 5-HT was delivered during the time indicated by the horizontal bar. The intracellular current was not significantly different for the paired and unpaired groups $(1.4 \pm 0.2 \mathrm{nA}$ for the paired group and $1.3 \pm 0.2 \mathrm{nA}$ for the unpaired group).

This excitability increase often leads to spontaneous firing when 5-HT is applied to unimpaled sensory neurons, so the number of action potentials during training is not adequately controlled. To counteract this effect, pairing experiments were performed in the presence of 1-3 $\mu \mathrm{M}$ tetrodotoxin (TTX), which has no effect on sensory neuron spike amplitude, yet is quite effective in reducing the excitability increase by nitrendipine. In control experiments we found that this amount of TTX, together with the same concentration of DMSO vehicle used to solubilize nitrendipine $(0.02$ or $0.10 \%)$, did not interfere with pairingspecific facilitation (data not shown).

Under these conditions, nitrendipine did not block the occurrence of pairing-specific facilitation. Neither $2 \mu \mathrm{M}(n=7)$ nor $10 \mu \mathrm{M}(n=6)$ nitrendipine appeared to eliminate pairingspecific facilitation. Because the results with either concentration were similar, data were pooled and are displayed in Figure 13. Overall, there was a marginal effect of pairing in the presence of nitrendipine $\left(F_{1,12}=3.17, p<0.05\right.$, one-tailed) and a significant pairing-by-trial interaction $\left(F_{3,34}=4.20, p<0.02\right)$, indicating that the paired effect differed over time. As previously reported (Edmonds et al., 1990), dihydropyridine blockade has no effect on basal synaptic transmission: EPSPs were normally depressed, to $79 \pm 5 \%$ of initial value after perfusing in TTX and DMSO $(n=31)$ versus $84 \pm 8 \%$ after adding TTX, DMSO, and nitrendipine $(n=22)$. Two observations suggest that the persistence of pairing-specific facilitation is not due to inefficacy of nitrendipine. First, the effect on sensory neuron excitability using either 2 or $10 \mu \mathrm{M}$ nitrendipine was very reliable, indicating that the drug is not highly labile. Second, we found in voltageclamp experiments that $2 \mu \mathrm{M}$ nitrendipine is about $80 \%$ as effective as $10 \mu \mathrm{M}$ at blocking the sustained $\mathrm{Ca}^{2+}$ current (not shown). These results indicate that $\mathrm{Ca}^{2+}$ influx through L-type channels is not necessary for the pairing effect, although the fact that the facilitation was somewhat less robust than in control experiments (Fig. 4) suggests these channels may make a partial contribution to the facilitation. Nonetheless, the failure of nitrendipine to block the associative effect implicates by elimination the dihydropyridine-insensitive channels as an important source of $\mathrm{Ca}^{2+}$ for pairing-specific facilitation.

\section{Discussion}

We have demonstrated that associative, activity-dependent synaptic facilitation can be induced in a reconstituted synapse in 
A

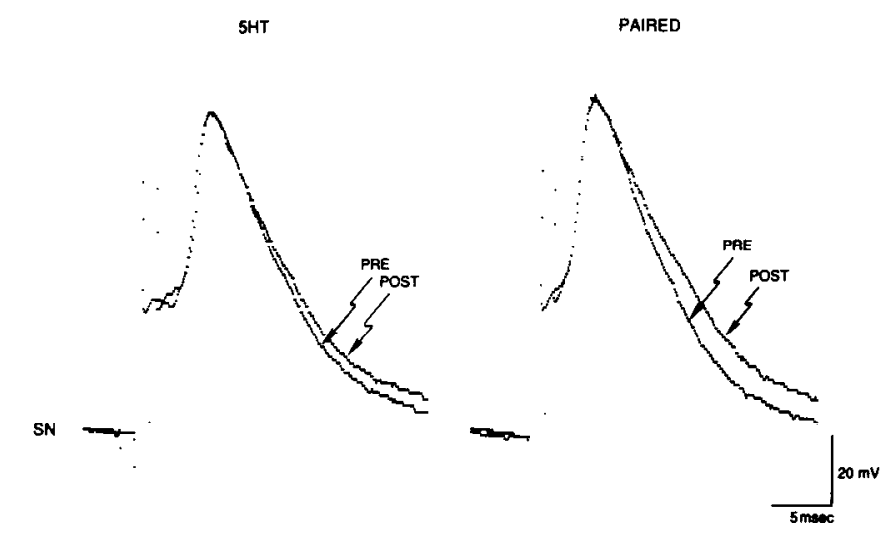

B

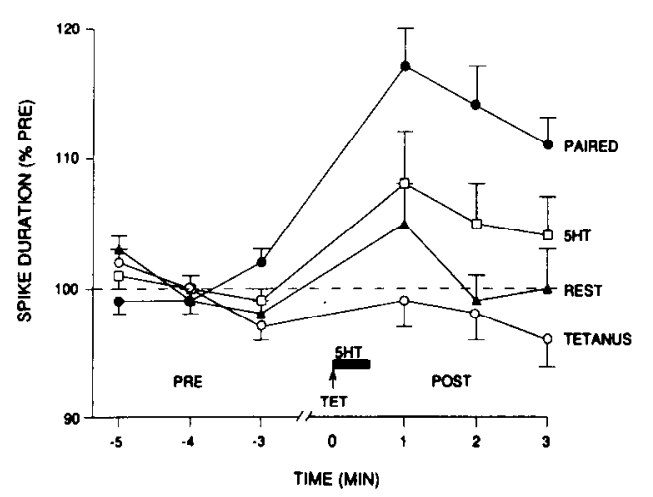

Figure 12. Pairing-specific increase in frequency-broadened spike width. $A$, The action potential in a sensory neuron at the end of a $1 \mathrm{sec}, 20$ $\mathrm{Hz}$ train before (PRE) and after (POST) training with either 5-HT alone or 5 -HT paired with a $2 \mathrm{sec}, 20 \mathrm{~Hz}$ tetanus. Paired training produced a larger increase in action potential duration that 5-HT alone. B, Group data ( $n=12$ per group). Paired training produced a significantly greater increase in spike width compared to 5-HT alone on the three trials following training. Tetanus alone produced a slight decrease compared to rest (a 3 min pause indicated by the break in the abscissa). Tetanus occurred at the arrow and 5-HT was delivered at the time indicated by the horizontal bar. Spike duration has been normalized to the average value on the three pretests, which was not significantly different for the four groups (7.1 $\pm 0.7 \mathrm{msec}$ for the paired group, $7.3 \pm 0.7$ for 5-HT alone, $8.4 \pm 0.8$ for tetanus alone, and $8.0 \pm 0.7$ for rest).

vitro. Pairing tetanus and 5-HT produces longer-lasting facilitation than either tetanus or 5-HT alone (Fig. 2). When normalized for the effect of homosynaptic depression, pairing-specific facilitation showed little decrement over the $20 \mathrm{~min}$ we monitored it (Fig. 3).

The relative durations of synaptic enhancement in our experiments in culture closely resemble those produced by activity alone (PTP), heterosynaptic modulation alone, and simultaneous activity + modulation in similar experiments in the ganglion (Walters and Byrne, 1985). In the animal, simultaneous activation of sensory neurons and their heterosynaptic modulators occurs in response to strong cutaneous activation, and the resulting enhanced facilitation likely contributes to behavioral site-specific sensitization (Walters, 1987). Here we took advantage of the reduced culture preparation to demonstrate that the enhanced facilitation also occurs when the onset of the tetanus precedes 5 -HT by $0.5 \mathrm{sec}$, but does not occur when tetanus is
PAIRING-SPECIFIC FACILITATION IN NITRENDIPINE

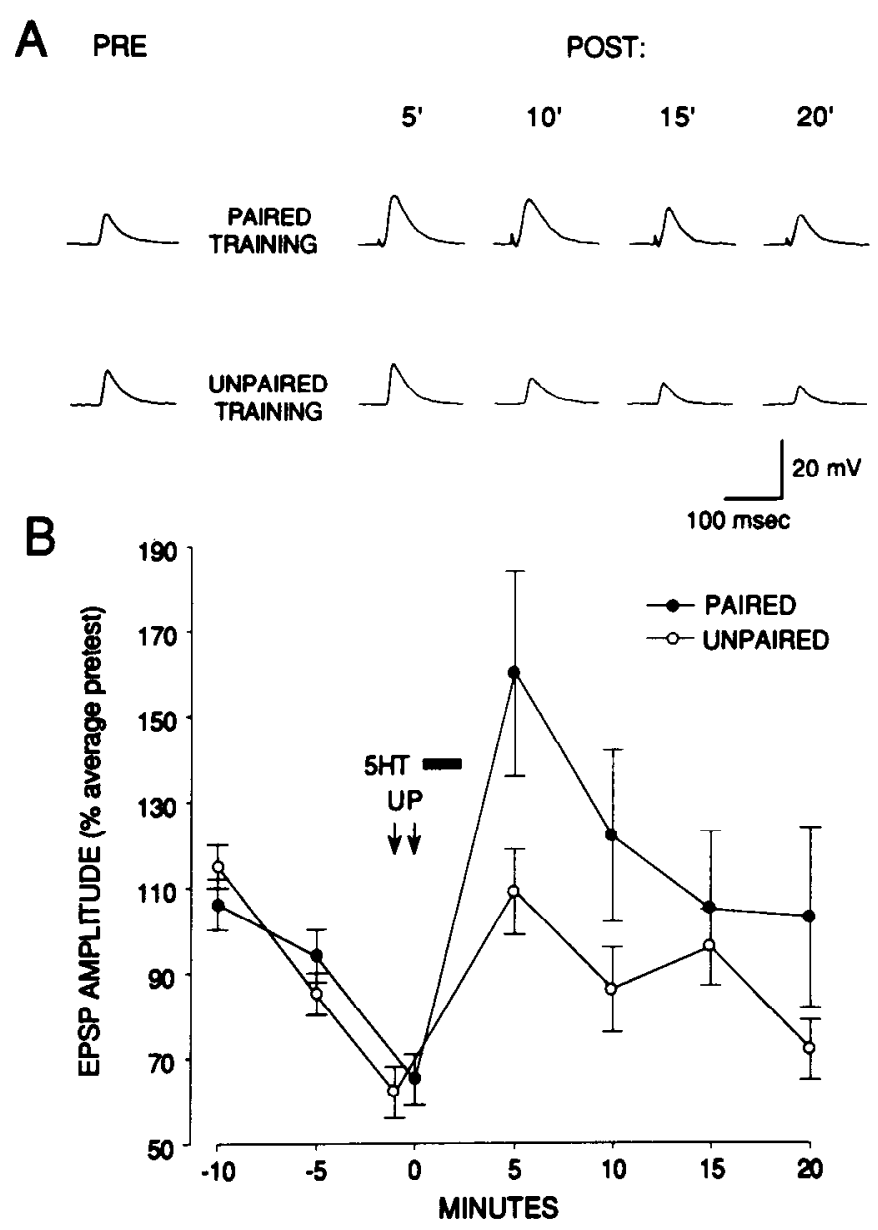

Figure 13. The L-type $\mathrm{Ca}^{3+}$ channel blocker nitrendipine does not block pairing-specific facilitation. $A$, EPSPs recorded in an LFS motor neuron in response to extracellular stimulation of paired (top) and unpaired (bottom) sensory neurons in the presence of $10 \mu \mathrm{M}$ nitrendipine $(0.1 \% \mathrm{DMSO})$, with $3 \mu \mathrm{M}$ TTX added to lower excitability. Despite the presence of nitrendipine, paired training significantly enhances facilitation compared to unpaired training. $B$, Group data from 13 experiments (six as in $A$, and seven using $2 \mu \mathrm{M}$ nitrendipine, $0.02 \%$ DMSO, $1 \mu \mathrm{M}$ TTX). Average EPSP amplitude before training was $14.9 \pm 3.0$ $\mathrm{mV}$ for paired cells and $14.0 \pm 2.8 \mathrm{mV}$ for unpaired cells.

delivered $1 \mathrm{~min}$ beforc 5-HT (Figs. 4, 5). This associative property makes the activity-dependent facilitation a good candidate for a cellular mechanism contributing to classical conditioning (Hawkins et al., 1983: Walters and Byrne, 1983), and thus our results support the idea that classical conditioning and sitespecific sensitization may share the same associative synaptic mechanism (Walters, 1987).

The finding that activity-dependent facilitation occurs in culture also demonstrates that it is intrinsic to the sensory-motor synapse. While it does not rule out a contribution of other circuit elements to associative facilitation in the ganglion, this finding demonstrates that such additional circuit elements are not necessary for the basic phenomenon. These results extend the range of synaptic plasticity that can be studied in culture to include not just homosynaptic and heterosynaptic plasticity, but conjunctive forms as well. This study also introduces a simple, one- 
trial protocol with all the technical advantages of cell culture to more easily analyze the mechanisms underlying pairing-specific facilitation.

Pairing-specific facilitation is mediated presynaptically, perhaps by action potential broadening

A major advantage of isolating single synapses in culture is that one can measure spontaneous transmitter release and be certain of its presynaptic source. We measured spontaneous release to look directly for changes in the release process during pairingspecific facilitation. The finding that unitary mEPSP or mEPSC size did not change significantly during pairing-specific facilitation (Fig. 8) indicates that the plasticity is mediated by a change in amount of transmitter released, rather than a change in postsynaptic responsiveness. Unitary amplitude did increase slightly following both paired and unpaired training, but this effect was not significant, nor is its magnitude sufficient to account for the magnitude of evoked FPSP facilitation. The finding of a presynaptic locus for pairing-specific facilitation is not surprising given that both components of this conjunctive plasticity - PTP and heterosynaptic facilitation - have previously been found to be presynaptically mediated in sensory neurons (Castellucci and Kandel, 1976; Dale and Kandel, 1990; Eliot, 1991).

Two mechanisms have been previously identified to participate in the presynaptic facilitation by 5-HT alone. The first involves CAMP-dependent broadening of the presynaptic action potential (Klein and Kandel, 1978), which enhances $\mathrm{Ca}^{2+}$ influx through voltage-gated channels in terminal regions (Eliot et al., 1993). A change in presynaptic spike width is necessary for facilitation of relatively nondepressed sensory neuron synapses (Hochner et al., 1986a). In contrast, when sensory neurons are depressed by repeated activation, the amount of rclcase is less sensitive to the duration of presynaptic depolarization, but 5-HT remains effective in facilitating the synapse. The exact mechanism by which 5 -HT induces this spike-independent enhancement of release is not known, but it has been termed "mobilization" because of the characteristic increase in EPSP slope it produces, as if the release sites are primed with vesicles to be rapidly released (Hochner et al., 1986b). While cAMP may also play a role in this mobilizing effect of 5-HT (Goldsmith and Abrams, 1991), the second process can be distinguished from the first process in that it involves protein kinase $\mathrm{C}$ (Braha et al., 1990; Ghirardi et al., 1992), which is also activated by 5-HT in sensory neurons (Sacktor and Schwartz, 1990; Sossin and Schwartz, 1992).

Our findings indicate that only the first of these two mechanisms contributes significantly to the one-trial, pairing-specific facilitation in culture. Using the same protocol, we find that sensory neuron spike width is enhanced in a pairing-specific manner (Fig. 12). The finding that sensory neuron excitability is also enhanced with paired training (Fig. 11) suggests that the broadening effect is due to cAMP-dependent closure of S-type $\mathrm{K}^{+}$channels (Klein et al., 1986; Baxter and Byrne, 1990). While both the excitability and spike-width effects in isolated sensory neurons appear to decay somewhat faster than the synaptic facilitation, the decay is probably accelerated by the frequent posttesting and the long depolarizing test commands (to measure excitability) or action potential trains (to measure spike width) used in these experiments. Sensory neuron impalement may also accelerate the decay of these associative biophysical changes, since it dramatically inhibits homosynaptic facilitation in cul- ture (Eliot, 1991). Thus, the true duration of pairing-specific increases in sensory neuron excitability and spike width is likely to be closer to that of the synaptic facilitation in culture, as was found previously for spike broadening and pairing-specific facilitation in the ganglion (Hawkins et al., 1983).

These results thus corroborate and extend earlier evidence that pairing-specific facilitation in the ganglion is due to an increase in action potential duration (Hawkins et al., 1983) and S-current downregulation (Hawkins and Abrams, 1984), and furthermore suggest that despite procedural differences, pairingspecific facilitation in culture and in the ganglion represent the same form of plasticity.

While our evidence supports a role of spike broadening, a number of findings argue against a role of the second process in the associative facilitation. First, the fact that we used predominantly naive or nondepressed synapses in these experiments suggests that the second process is unlikely to be involved, since this mechanism is defined by its role in reversing synaptic depression (Hochner et al., 1986h; Ghirardi et al., 1992). A second piece of evidence is our finding that pairing-specific facilitation can be induced as well using the modulatory peptide SCP as it is using 5-HT (Fig. 3). Like 5-HT, SCP increases CAMP levels in sensory neurons, broadens the presynaptic action potential, and facilitates sensory-motor synaptic transmission (Abrams et al., 1984). However, unlike 5-HT, SCP does not activate the second process (Schacher et al., 1990). A third piece of evidence is that the inhibitor $\mathrm{H} 7$, which blocks the component of the second process mediated by protein kinase $C$, did not prevent pairing-specific facilitation (Fig. 9). Like the facilitation of depressed synapses, the enhancement of spontaneous release frequency by $5-\mathrm{HT}$ is mediated by protein kinase $\mathrm{C}$ (Braha et al., 1990; Ghirardi et al., 1992). Thus, the last piece of evidence argues particularly strongly against a rolc of the sccond processthe finding that the associative facilitation is not accompanied by a pairing-specific enhancement of spontaneous release frequency (Fig. 7). The fact that spontaneous release frequency does not remain elevated during the sustained period of evoked facilitation tends generally to rule out any facilitatory mechanism that is independent of the action potential. However, it was recently found that associative facilitation in the ganglion produces an increase in EPSP slope, which is thought to reflect predominantly the second process (G. A. Clark, R. D. Hawkins, and E. R. Kandel, unpublished observations). Thus, it remains possible that pairing-specific facilitation involves a protein kinase $\mathrm{C}$-independent component of mobilization not reflected in spontaneous release.

\section{Conclusion}

We have provided evidence that associative synaptic facilitation occurs in isolated synapses in culture and that it is presynaptically mediated by mechanisms unique to evoked transmitter release. Our findings indicate that $\mathrm{CAMP}$-dependent kinase, and not protein kinase $C$, is critically involved in pairing-specific facilitation. While $\mathrm{Ca}^{2+}$ influx is probably necessary to induce the associative effect (Abrams, 1985), influx through dihydropyridine-sensitive channels is required neither for its induction nor its expression. These results suggest that a pairing-specific increase in presynaptic action potential width contributes to the associative facilitation by enhancing $\mathrm{Ca}^{2+}$ influx through the dihydropyridine-insensitive channels underlying transmitter release. 


\section{References}

Abrams TW (1985) Activity-dependent presynaptic facilitation: an associative mechanism in Aplysia. Cell Mol Neurobiol 5: 123-145.

Abrams TW, Kandel ER (1988) Is contiguity detection in classical conditioning a system or a cellular property? Learning in Aplysia suggests a possible molecular site. Trends Neurosci 1 1:128-135.

Abrams TW, Castellucci VF, Camardo JS, Kandel ER, Lloyd PE (1984) Two endogenous neuropeptides modulate the gill and siphon withdrawal reflex in Aplysia by presynaptic facilitation involving cAMPdependent closure of a serotonin-sensitive potassium channel. Proc Natl Acad Sci USA 81:7956-7960.

Abrams TW, Karl KA, Kandel ER (1991) Biochemical studies of stimulus convergence during classical conditioning in Aplysia: dual regulation of adenylate cyclase by $\mathrm{Ca}^{2+} /$ calmodulin and transmitter. J Neurosci 11:2655-2665.

Bank M, Schacher S (1992) Segregation of presynaptic inputs on an identified target neuron in vitro: structural remodeling visualized over time. J Neurosci 12:2960-2972.

Barrionuevo G, Brown TH (1983) Associative long-term potentiation in hippocampal slices. Proc Natl Acad Sci USA 80:7347-7351.

Baxter DA, Byrne JH (1990) Differential effects of cAMP and serotonin on membrane current, action potential duration, and excitability in somata of pleural sensory neurons of Aplysia. J Neurophysiol 64:978-990.

Braha O, Dale N, Hochner B, Klein M, Abrams TW, Kandel ER, Klein $M$ (1990) Second messengers involved in the two processes of presynaptic facilitation that contribute to sensitization and dishabituation in Aplysia sensory neurons. Proc Natl Acad Sci USA 87:20402044.

Braha O, Edmonds B, Sacktor T, Kandel ER (1993) The contributions of protein kinase $A$ and protein kinase $\mathrm{C}$ to the actions of $5-\mathrm{HT}$ on the L-type $\mathrm{Ca}^{2+}$ current of the sensory neurons in Aplysia. J Neurosci 13:1839-1851.

Brunelli M, Castellucci V, Kandel ER (1976) Synaptic facilitation and behavioral sensitization in Aplysia: possible role of serotonin and cyclic AMP. Science 194:1178-1181.

Buonomano DV, Byrne JH (1990) Long-term synaptic changes produced by a cellular analog of classical conditioning in Aplysia. Science 249:420-423.

Byrne JH (1987) Cellular analysis of associative learning. Physiol Rev $67: 329-439$

Carew TJ, Walters ET, Kandel ER (1981) Classical conditioning in a simple withdrawal reflex in Aplysia californica. J Neurosci 1:14261437

Carew TJ, Hawkins RD, Kandel ER (1983) Differential classical conditioning of a defensive withdrawal reflex in Aplysia californica. Science 219:397-400.

Castellucci V, Kandel ER (1976) Presynaptic facilitation as a mechanism for behavioral sensitization in Aplysia. Science 194:1176-1178.

Castellucci VF, Nairn A, Greengard P, Schwartz JH, Kandel ER (1982) Inhibitor of adenosine $3^{\prime}: 5^{\prime}$-monophosphate-dependent protein kinase blocks presynaptic facilitation in Aplysia. J Neurosci 2:16731681 .

Cline HT (1991) Activity-dependent plasticity in the visual systems of frogs and fish. Trends Neurosci 14:104-111.

Collingridge GL, Singer W (1990) Excitatory amino acid receptors and synaptic plasticity. Trends Pharmacol Sci 11:290-296.

Dale N, Kandel ER (1990) Facilitatory and inhibitory transmitters modulate spontaneous transmitter release at cultured Aplysia sensorimotor synapses. J Physiol (Lond) 42 1:203-222.

Edmonds B, Klein M, Dale N, Kandel ER (1990) Contributions of two types of calcium channels to synaptic transmission and plasticity. Science 250:1142-1147.

Eisenstadt M, Goldman JE, Kandel ER, Koike H, Koester J, Schwartz JH (1973) Intrasomatic injection of radioactive precursors for studying transmitter synthesis in identified neurons of Aplysia californica. Proc Natl Acad Sci USA 70:3371-3375.

Eliot LS (1991) Presynaptic modulation of Aplysia sensory-to-motor neuron synapses in culture. PhD thesis, Columbia University.

Eliot LS, Dudai Y, Kandel ER, Abrams TW (1989) $\mathrm{Ca}^{2+} /$ calmodulin sensitivity may be common to all forms of neural adenylate cyclase. Proc Natl Acad Sci USA 86:9564-9568.

Eliot LS, Kandel ER, Hawkins ER (1990) Activity-dependent mod- ulation of spontaneous transmitter release in single cultured Aplysia sensorimotor neuron synapses. Soc Neurosci Abstr 16:1013.

Eliot LS, Kandel ER, Siegelbaum SA, Blumenfeld H (1993) Imaging terminals of Aplysia sensory neurons demonstrates role of enhanced $\mathrm{Ca}^{2+}$ influx in presynaptic facilitation. Nature 361:634-637.

Ghirardi M, Braha O, Hochner B. Montarolo PG, Kandel ER, Dale N (1992) Roles of PKA and PKC in facilitation of evoked and spontaneous transmitter release at depressed and nondepressed synapses in Aplysia sensory neurons. Neuron 9:470-489.

Glanzman DL, Mackey SL, Hawkins RD, Dyke AM, Lloyd PE, Kandel ER (1989) Depletion of serotonin in the nervous system of Aplysia reduces the bchavioral enhancement of gill withdrawal as well as the heterosynaptic facilitation produced by tail shock. J Neurosci 9:4200_ 4213.

Goldsmith BA, Abrams TW (1991) Reversal of synaptic depression by serotonin at Aplysia sensory neuron synapses involves activation of adenylate cyclase. Proc Natl Acad Sci USA 88:9021-9025.

Goldsmith BA, Abrams TW (1992) cAMP modulates multiple K currents, increasing spike duration and excitability in Aplysia sensory neurons. Proc Natl Acad Sci USA 89:11481-11485.

Hamill OP, Marty A, Neher E, Sakmann B, Sigworth FJ (1981) Improved patch-clamp techniques for high-resolution current recording from cells and cell-free membrane patches. Pfluegers Arch 391:85100.

Hawkins RD (1991) Cell biological studies of conditioning in Aplysia. In: Neurobiology of learning, emotion and affect (Madden J, cd), pp 3-28. New York: Raven.

Hawkins RD, Abrams TW (1984) Evidence that activity-dependent facilitation underlying classical conditioning in Aplysia involves modulation of the same ionic current as normal presynaptic facilitation. Soc Neurosci Abstr 10:268.

Hawkins RD, Schacher S (1989) Identified facilitator neurons L29 and L28 are excited by cutaneous stimuli used in dishabituation, sensitization, and classical conditioning of Aplysia. J Neurosci 9:42364245.

Hawkins RD, Castellucci VF, Kandel ER (1981) Interneurons involved in mediation and modulation of gill-withdrawal reflex in $\mathrm{Aply}$ sia. II. Identified neurons produce heterosynaptic facilitation contributing to behavioral sensitization. J Neurophysiol 45:315-326.

Hawkins RD, $\Lambda$ brams TW, Carew TJ, Kandel ER (1983) A ccllular mechanism of classical conditioning in Aplysia: activity-dependent amplification of presynaptic facilitation. Science 219:400-405.

Hawkins RD, Kandel ER, Siegelbaum SA (1993) Learning to modulate transmitter release: themes and variations in synaptic plasticity. Annu Rev Neurosci 16:625-665.

Hickie C, Walters ET (1990) Identified central motor neurons are necessary for directional siphon responses in Aplysia. Soc Neurosci Abstr 16:19.

Hochner B, Kandel ER (1992) Modulation of a transient K+ current in the pleural sensory neurons of Aplysia by serotonin and cAMP: implications for spike broadening. Proc Natl Acad Sci USA 89:1147611480.

Hochner B, Klein M, Schacher S, Kandel ER (1986a) Action-potential duration and the modulation of transmitter release from the sensory neurons of Aplysia in presynaptic facilitation and behavioral sensitization. Proc Natl Acad Sci USA 83:8410-8414.

Hochner B, Klein M, Schacher S, Kandel ER (1986b) Additional component in the cellular mechanism of presynaptic facilitation contributes to behavioral dishabituation in Aplysia. Proc Natl Acad Sci USA 83:8794-8798.

Ito M, Sakurai M, Tongroach P (1982) Climbing fibre induced depression of both mossy fiber responsiveness and glutamate sensitivity of cerebellar Purkinje cells. J Physiol (Lond) 324:113-134.

Klein M, Kandel ER (1978) Presynaptic modulation of voltage-dependent $\mathrm{Ca}^{2+}$ current: mechanism for behavioral sensitization in Aplysia californica. Proc Natl Acad Sci USA 75:3512-3516.

Klein M, Camardo J, Kandel ER (1982) Serotonin modulates a specific potassium current in the sensory neurons that show presynaptic facilitation in Aplysia. Proc Natl Acad Sci USA 79:5713-5717.

Klein M, Hochner B, Kandel ER (1986) Facilitatory transmitters and cAMP can modulate accommodation as well as transmitter release in Aplysia sensory neurons: evidence for parallel processing in a single cell. Proc Natl Acad Sci USA 83:7994-7998.

Levy WB, Steward O (1979) Synapses as associative memory elements in the hippocampal formation. Brain Res 175:233-245. 
Mackey SL, Kandel ER, Hawkins RD (1989) Identified serotonergic neurons LCB1 and RCB1 in the cerebral ganglia of Aplysia produce presynaptic facilitation of siphon sensory neurons. J Neurosci 9:42274235.

Mercer AR, Emptage NJ, Carew TJ (1991) Pharmacological dissociation of modulatory effects of serotonin in Aplysia sensory neurons. Science 254:1811-1813.

Montarolo PG, Goelet P, Castellucci VF, Morgan J, Kandel ER, Schacher $S$ (1986) A critical period of macromolecular synthesis in longterm heterosynaptic facilitation in Aplysia. Science 234:1249-1254.

Montarolo PG, Kandel ER, Schacher S (1988) Long-term heterosynaptic inhibition in Aplysia. Nature 333:171-174.

Nerbonne JM, Gurney AM (1987) Blockade of $\mathrm{Ca}^{2+}$ and $\mathrm{K}^{+}$currents in bag cell neurons of Aplysia californica by dihydropyridine $\mathrm{Ca}^{2+}$ antagonists. J Neurosci 7:882-893.

Ocorr KA, Walter ET, Byrne JH (1985) Associative conditioning analog selectively increases cAMP levels of tail sensory neurons in $A p l y$ sia. Proc Natl Acad Sci USA 82:2548-2552.

Rayport SG, Schacher S (1986) Synaptic plasticity in vitro: cell culture of identified Aplysia neurons mediating short-term habituation and sensitization. J Neurosci 6:759-763.

Sacktor TC, Schwartz JH (1990) Sensitizing stimuli cause translocation of protein kinase $C$ in Aplysia sensory neurons. Proc Natl Acad Sci USA 87:2036-2039.

Schacher S, Montarolo PG, Kandel ER (1990) Selective short- and long-term effects of serotonin, small cardioactive peptide, and tetanic stimulation on sensorimotor synapses of Aplysia in culture. J Neurosci 10:3286-3294.

Shuster MJ, Camardo JS, Siegelbaum SA, Kandel ER (1985) Cyclic
AMP-dependent protein kinase closes the serotonin-sensitive $\mathrm{K}$ channels of Aplysia sensory neurones in cell-free membrane patches. Nature 313:392-395.

Siegelbaum SA, Camardo JS, Kandel ER (1982) Serotonin and cyclic AMP close single $\mathrm{K}^{+}$channels in Aplysia sensory neurons. Nature 299:413-417.

Small SA, Kandel ER, Hawkins RD (1989) Activity-dependent enhancement of presynaptic inhibition in Aplysia sensory neurons. Science 243:1603-1606.

Sossin WS, Schwartz JH (1992) Selective activation of $\mathrm{Ca}^{2+}$-activated PKCs in Aplysia neurons by 5-HT. J Neurosci 12:1160-1168.

Stanton PK, Sejnowski TJ (1989) Associative long-term depression in the hippocampus induced by hebbian covariance. Nature 339 215-218.

Walters ET (1987) Multiple sensory neuronal correlates of site-specific sensitization in Aplysia. J Neurosci 7:408-417.

Walters ET, Byrne JH (1983) Associative conditioning of single sensory neurons suggests a cellular mechanism for learning. Science 219 . 405-408.

Walters ET, Byrne JH (1985) Long-term enhancement produced by activity-dependent modulation of Aplysia sensory neurons. J Neurosci 5:662-672.

Walters ET, Byrne JH, Carew TJ, Kandel ER (1983) Mechanoafferent neurons innervating tail of Aplysia: 1. Response properties and synaptic connections. J Neurophysiol 50:1522-1542.

Yovell Y, Abrams TW (1992) Temporal asymmetry in activation of Aplysia adenylyl cyclase by calcium and transmitter may explain temporal requirements of conditioning. Proc Natl Acad Sci USA 89: $6526-6530$. 